\title{
Spatial distribution of star formation related to ionized regions throughout the inner Galactic plane
}

\author{
P. Palmeirim ${ }^{1,2}$, A. Zavagno ${ }^{1}$, D. Elia ${ }^{3}$, T. J. T. Moore ${ }^{4}$, A. Whitworth ${ }^{5}$, P. Tremblin 6 , A. Traficante ${ }^{3}$, M. Merello ${ }^{3}$,
} D. Russeil ${ }^{1}$, S. Pezzuto ${ }^{3}$, L. Cambrésy ${ }^{7}$, A. Baldeschi ${ }^{3}$, M. Bandieramonte $^{8}$, U. Becciani $^{9}$, M. Benedettini ${ }^{3}$, C. Buemi ${ }^{9}$, F. Bufano ${ }^{9}$, A. Bulpitt ${ }^{10}$, R. Butora ${ }^{11}$, D. Carey ${ }^{10}$, A. Costa ${ }^{9}$, L. Deharveng ${ }^{1}$, A. Di Giorgio ${ }^{3}$, D. Eden ${ }^{4}$, A. Hajnal ${ }^{12}$, M. Hoare ${ }^{10}$, P. Kacsuk ${ }^{12}$, P. Leto ${ }^{9}$, K. Marsh ${ }^{5}$, P. Mège ${ }^{1}$, S. Molinari $^{3}$, M. Molinaro ${ }^{11}$, A. Noriega-Crespo ${ }^{13}$, E. Schisano ${ }^{3}$, E. Sciacca ${ }^{9}$, C. Trigilio ${ }^{9}$, G. Umana ${ }^{9}$, and F. Vitello ${ }^{9}$

(Affiliations can be found after the references)

Received 26 October 2016 / Accepted 17 May 2017

\begin{abstract}
We present a comprehensive statistical analysis of star-forming objects located in the vicinities of 1360 bubble structures throughout the Galactic plane and their local environments. The compilation of $\sim 70000$ star-forming sources, found in the proximity of the ionized (HII) regions and detected in both Hi-GAL and GLIMPSE surveys, provided a broad overview of the different evolutionary stages of star-formation in bubbles, from prestellar objects to more evolved young stellar objects (YSOs). Surface density maps of star-forming objects clearly reveal an evolutionary trend where more evolved star-forming objects (Class II YSO candidates) are found spatially located near the center, while younger star-forming objects are found at the edge of the bubbles. We derived dynamic ages for a subsample of $182 \mathrm{HII}$ regions for which kinematic distances and radio continuum flux measurements were available. We detect approximately $80 \%$ more star-forming sources per unit area in the direction of bubbles than in the surrounding fields. We estimate the clump formation efficiency (CFE) of Hi-GAL clumps in the direction of the shell of the bubbles to be $\sim 15 \%$, around twice the value of the CFE in fields that are not affected by feedback effects. We find that the higher values of CFE are mostly due to the higher CFE of protostellar clumps, in particular in younger bubbles, whose density of the bubble shells is higher. We argue that the formation rate from prestellar to protostellar phase is probably higher during the early stages of the (H II ) bubble expansion. Furthermore, we also find a higher fraction of massive YSOs (MYSOs) in bubbles at the early stages of expansion ( $<2$ Myr) than older bubbles. Evaluation of the fragmentation time inside the shell of bubbles advocates the preexistence of clumps in the medium before the bubble expansion in order to explain the formation of MYSOs in the youngest $\mathrm{H}$ II regions (<1 Myr), as supported by numerical simulations. Approximately $23 \%$ of the Hi-GAL clumps are found located in the direction of a bubble, with $15 \%$ for prestellar clumps and $41 \%$ for protostellar clumps. We argue that the high fraction of protostellar clumps may be due to the acceleration of the star-formation process cause by the feedback of the (HII) bubbles.
\end{abstract}

Key words. HII regions - stars: formation - stars: massive - infrared: ISM - submillimeter: ISM

\section{Introduction}

Thanks to ground- and space-based surveys of the Galactic plane, a new picture of the interstellar medium has emerged. In particular, Spitzer images at $8 \mu \mathrm{m}$ and $24 \mu \mathrm{m}$ revealed an almost ubiquitous presence of bubble structures seen throughout the entire Galactic plane (Churchwell et al. 2006, 2007). Anderson et al. (2011) have found that half of the HII regions have a bubble morphology. H II regions are generated by massive stars that ionize the surrounding medium, causing it to expand isotropically. The $8 \mu \mathrm{m}$ emission appears in the Spitzer images as a ring-like structure caused by the emission of polycyclic aromatic hydrocarbon $(\mathrm{PAH})$ molecules that are concentrated in the shell of the bubble, which are excited by the UV emission of the ionizing source(s) (Leger \& Puget 1984; Draine \& Li 2007; Tielens 2008). On the other hand, the $24 \mu \mathrm{m}$ emission is typically seen to radiate in the inner part of the bubbles, but its origin is still unclear (see Deharveng et al. 2010). It is normally attributed to very small dust grains that can survive at shorter distances from the ionizing sources (Desert et al. 1990), but some evidence also suggests that $24 \mu \mathrm{m}$ emission may be caused by large dust grains in thermal equilibrium (Cesarsky et al. 2000).

As bubbles expand, they interact with the surrounding cold molecular medium, which may trigger star formation. Three main physical mechanisms are considered when the triggering of star-formation is discussed: the collect and collapse (C\&C; Elmegreen \& Lada 1977), the radiation-driven implosion (RDI; Deharveng et al. 2010), and the enhancement of preexisting density substructures and subsequent global implosion (EDGI; Walch et al. 2015). The C\&C mechanism causes the cold gas and dust to be swept up by the supersonic expansion of an $\mathrm{H}$ II region and to be compressed in between the ionization and shock fronts. As a result, the compressed collected material can reach high densities, become gravitationally unstable, and fragment into massive dense clumps (Whitworth et al. 1994; Deharveng et al. 2005; Zavagno et al. 2010). Conversely, the RDI mechanism arises from the interaction of the ionizing radiation with the turbulent surrounding medium. This process is responsible for the triggering of preexisting condensations of dense gas, as well as for the formation of pillar structures (Hester et al. 1996; Tremblin et al. 2012). The EDGI mechanism describes the expansion of the HII regions in a fractal medium, taking the preexistence of dense structures into account, which through a hybrid interaction of the $\mathrm{C} \& \mathrm{C}$ and $\mathrm{RDI}$ processes trigger on much shorter timescales $(<1 \mathrm{Myr})$, as demonstrated in numerical simulations (Walch et al. 2012, 2013, 2015).

Star formation is indeed observed at the edges of ionized H II regions, as shown by several observational studies 
(Zavagno et al. 2007, 2010; Deharveng et al. 2010; Samal et al. 2014; Liu et al. 2016). In particular, Thompson et al. (2012) and Kendrew et al. (2012) made a detailed statistical analysis using Spitzer bubbles, which revealed that massive young stellar objects (YSOs) identified in the Red MSX Source survey (Urquhart et al. 2008) were typically observed at the edge of the bubbles, while intrinsically red sources from the Robitaille et al. (2008) catalog exhibit a flat distribution inside the bubbles. Since the Robitaille et al. (2008) sample contained more evolved objects, Thompson et al. suggested that this may be due to an evolutionary gradient across the bubble. Moreover, about $\sim 25 \%$ of MYSOs throughout the Galactic plane are located at the edges of H II regions, which means that around one quarter of MYSOs formed in the Milky Way might have been triggered by H II regions.

Although several results suggest that triggered star formation may be occurring, the exact relation between the presence of an $\mathrm{H}$ II region, its expansion, and star formation observed in its vicinity is difficult to establish (Dale et al. 2015). Nevertheless, the link between $\mathrm{H}$ II regions and triggered star-formation may be better addressed by studying several triggering indicators in a large sample of $\mathrm{H}$ II regions.

In the present work, we aim to exploit the combined information of new-generation surveys of the Galactic plane to deliver a global understanding of the star formation in $\mathrm{H}$ II regions and their local environments for a large sample of 1360 bubbles that are located at various places throughout the Galactic plane. In particular, Herschel data from the Herschel Infrared Galactic plane survey (Hi-GAL; Molinari et al. 2010, 2016) allow us to detect star-forming objects in earlier evolutionary stages and view the cold dust in their surroundings. Combined with the detection of YSO at a more advance evolutionary stage using the Spitzer-GLIMPSE source catalog, we wish to obtain a broad overview of the different evolutionary stages of star formation in bubbles.

This paper is organized as follows. In Sect. 2 we describe the observations and their respective data reduction procedures, and present the sample of (HII) bubbles we used that are located in the inner Galactic plane. Section 3 presents the sample selection and classification of GLIMPSE YSOs and Hi-GAL clumps found in the vicinities of (HII) bubbles. Section 4 presents the results, which are discussed in Sect. 5. Summary and conclusions are presented in Sect. 6.

\section{Observations and data reduction}

\subsection{Herschel Hi-GAL observations}

We made use of Herschel observations (Pilbratt et al. 2010) of the inner part of the Galactic plane $\left(68^{\circ} \geq l \geq-70^{\circ}\right.$ and $\left.|b| \leq 1^{\circ}\right)$, which were obtained as part of the Herschel Infrared Galactic plane survey (Hi-GAL; Molinari et al. 2010, 2016). The Galactic plane was covered by individual observations of $\sim 2.2$ tiles that were mapped in two orthogonal scan directions at $60^{\prime \prime} \mathrm{s}^{-1}$, from which we simultaneously obtained PACS (Poglitsch et al. 2010) $70 \mu \mathrm{m}$ and $160 \mu \mathrm{m}$ and SPIRE (Griffin et al. 2010) at $250 \mu \mathrm{m}, 350 \mu \mathrm{m}$, and $500 \mu \mathrm{m}$ images using the parallel mode of Herschel.

The data reduction was performed using the ROMAGAL data-processing code for both PACS and SPIRE (see Traficante et al. 2011, for details). The raw data were first processed up to level 0.5 using standard steps in the pipeline of the Herschel interactive processing environment (HIPE; Ott 2010). Post-processing was carried out with a dedicated pipeline written by the Hi-GAL Consortium (see Molinari et al. 2016, for details), which removes glitches and slow thermal drifts. The final PACS and SPIRE maps were produced by a map-making algorithm based on generalized least squares (GLS; Natoli et al. 2001).

Zero-level offsets were added to the Herschel images based on the comparison between the Herschel data with IRIS (Improved Reprocessing of the IRAS Survey) all-sky maps and Planck data at comparable wavelengths (cf. Bernard et al. 2010; Molinari et al. 2016).

\subsection{Galactic plane bubble sample}

For this work we made use of the Milky Way Project $\left(\mathrm{MWP}^{1}\right)$ catalog (Simpson et al. 2012). This catalog was obtained by compressing a very large sample of visual measurements on the Spitzer-GLIMPSE $8 \mu \mathrm{m}$ and $24 \mu \mathrm{m}$ maps, performed by over 35000 independent volunteers that are part of the large community of the "citizen scientists" Zooniverse ${ }^{2}$ project. The MWP bubbles were classified into two separate categories, small and large catalogs, based on their sizes. Since the MWP small bubble catalog contains bubbles that are not well resolved in the Spitzer images, we opted to select bubbles from the large catalog, which provides more reliable statistical measurements.

To ensure that the selected MWP bubbles were resolved in the Herschel maps, we opted to only select from the catalog bubbles with effective radius ${ }^{3}\left(R_{\text {eff }}\right)$ larger than $72^{\prime \prime}$ (approximately twice the full-width at half maximum $(F W H M)$ of the Herschel SPIRE $500 \mu \mathrm{m}$ beam). This led to a total sample of 1360 bubbles and covers the $|l|<65^{\circ}$ and $|b|<1^{\circ}$ range of the Galactic plane.

\section{Source detection and classification}

\subsection{Hi-GAL prestellar and protostellar objects}

To probe recent star formation activity in the vicinities of bubbles, we made use of the physical Hi-GAL source catalog (Elia et al. 2017). The Hi-GAL sources were first extracted from the Hi-GAL PACS and SPIRE images using the CuTeX photometry code (Molinari et al. 2010), from which we generated an individual photometric catalog for each band for the inner Galactic plane, as described in Molinari et al. (2016). The singleband catalogs were then compiled into a band-merged catalog following Elia et al. (2010, 2013). A final sample of 100922 sources with detection in at least three consecutive bands between $160 \mu \mathrm{m}$ and $500 \mu \mathrm{m}$ were considered eligible for the fit of a modified blackbody to their spectral energy distributions (SEDs) and were included in the Hi-GAL physical cata$\log$, with their respective derived physical properties. Moreover, sources were classified according to their evolutionary stages following the same approach as described in Elia et al. (2013). A $70 \mu \mathrm{m}$ counterpart was used to distinguish protostellar and starless sources, and the latter were further classified into gravitationally unbound and bound (prestellar) sources, based on the mass threshold inferred by Larson's third law. Potential biases due to issues such as distances, multiplicities, and saturations are addressed in Elia et al. (2017).

For the present work we selected from the catalog all prestellar (starless and gravitationally bound) and protostellar (with a

\footnotetext{
1 http://www.milkywayproject.org

2 http://zooinverse.org

$3 R_{\text {eff }}$ is defined in the MWP catalog as the mean between the geometrically averaged semi-minor and semi-major axis of the inner and outer ellipses of the bubble.
} 
$70 \mu \mathrm{m}$ counterpart) objects that were found within four times the effective radius ( $\left.<4 R_{\text {eff }}\right)$ of a bubble. This led to a total sample of 25911 prestellar and 14918 protostellar sources that were used in our study. Since the large majority of the detected sources in the Galactic plane are located at distances between $2-15 \mathrm{kpc}$ (see text bellow), we expect their physical sizes to be greater then 0.1 pc. Thus, we henceforth define them as clumps (e.g., Bergin \& Tafalla 2007).

However, we would like to note that considering detection at $70 \mu \mathrm{m}$ as a selection criteria for the protostellar clumps located in the vicinity of $\mathrm{H}$ II regions can cast some doubt on their reliability. The bulk of the emission of H II regions is caused by the photodissociation region (PDR) formed at the edges that strongly emit at $70 \mu \mathrm{m}$ (Paladini et al. 2012). Owing to the nebular morphology of the PDR, it may be possible to find some compact knots that can led to a false classification of a protostellar clump that can lead to the classification of a prestellar clump as a protostellar clump (e.g., Gaczkowski et al. 2013). To address this issue and the reliability of our protostellar clump sample, we used the information of mid-infrared (MIR) counterparts that is available in the Hi-GAL catalog, since protostellar sources also emit in the MIR, but the effect from the PDR is considerably fainter at this wavelength. For each Hi-GAL source a MIR counterpart was searched for at 24,22 , and $21 \mu \mathrm{m}$ in the respective MIPSGAL (Gutermuth \& Heyer 2015), WISE (Wright et al. 2010), and MSX (Egan et al. 2003) data, when available (see Elia et al. 2017, for further details). Using this information, we find that $\sim 90 \%$ of our protostellar clump sample has at least one counterpart measurement at one of the aforementioned wavelengths in the MIR. Therefore, the level of potential contamination that is due to PDR is not considered in the further analysis.

\subsection{GLIMPSE YSO source classification}

As protostellar objects accrete most of their surrounding envelope, they evolve into a YSO, at which point outflows start to further clear the envelope, shifting the peak emission toward mid-infrared wavelengths. Spitzer, with its optimized wavelength coverage to detect and characterize the different evolutionary stages of YSOs, has been used extensively in previous works (e.g., Allen et al. 2004; Evans et al. 2009; Heiderman et al. 2010; Rebull et al. 2010).

In this work, the initial selection of YSO candidates was based on the color criteria of IRAC photometric measurements made available in the GLIMPSE Source Catalog (I + II + 3D) (Spitzer Science Center 2009), following a similar approach as Gutermuth et al. (2008). Only sources with IRAC magnitudes brighter than 14.2, 14.1, 11.9, and $10.8 \mathrm{mag}$ at 3.6, 4.5, 5.8, and $8.0 \mu \mathrm{m}$ were considered to ensure a detection reliability above $98 \%$ in all IRAC bands, based on the incompleteness levels inferred in the GLIMPSE I Assurance Quality Document ${ }^{4}$. In order to preserve sources with good-quality photometry, we avoided detections with an adjacent companion by verifying that their close source flag (csf) 5 was equal to zero and that the magnitude errors were lower than 0.2 mag (Robitaille et al. 2008).

For the selection criteria of YSO candidates we followed the Gutermuth et al. (2009) complementary analysis of the Bootes field of IRAC data (Stern et al. 2005), which allows removing potential contamination of star-forming galaxies and active galactic nuclei (AGNs). In practice, YSO candidates were

\footnotetext{
4 http://www . astro.wisc. edu/glimpse/GQA-master.pdf

5 Flag sources in the Archive Catalog with a companion within $3^{\prime \prime}$.
}

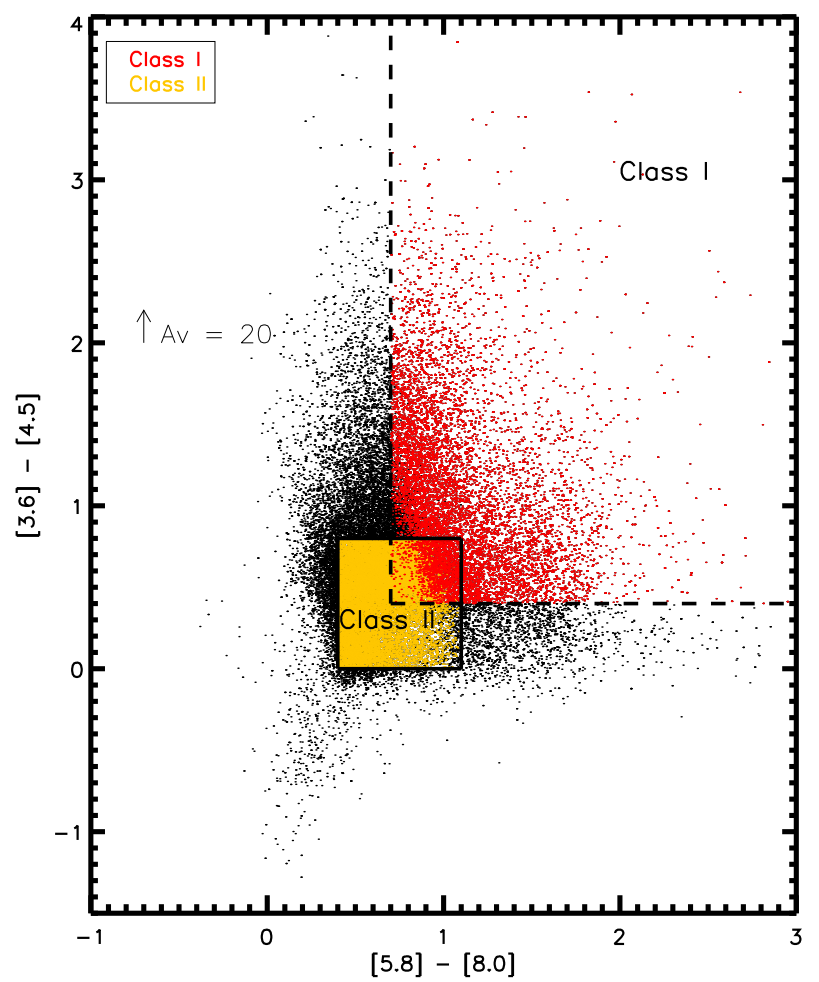

Fig. 1. IRAC color-color diagram of 91026 YSO candidates located at less than four times the effective radius $\left(R_{\mathrm{eff}}\right)$ of the 1360 bubbles. The solid and dashed lines represent the respective boundaries of Class I and II sources, following Allen et al. (2004). The red and yellow dots represent the Class I (10 694) and Class II (18 209) YSO candidates selected based on both their spectral indices and position on the diagram, respectively. The reddening vector of $A_{\mathrm{V}}=20 \mathrm{mag}$ was derived from the extinction law of Indebetouw et al. (2005).

selected by applying the following color conditions:

$[4.5]-[8.0]>0.5$,

$[3.6]-[5.8]>0.35$,

$[3.6]-[5.8] \leq 3.5 \times([4.5]-[8.0])-1.25$.

These selection criteria were applied to all GLIMPSE sources found within $4 \times R_{\text {eff }}$ of a bubble, which led to a total of 91026 YSO candidates.

The selected YSO candidates were further classified into different evolutionary stages according to their infrared spectral in$\operatorname{dex} \alpha_{\text {IRAC }}=\mathrm{d} \log \left(v F_{v}\right) / \mathrm{d} \log (v)$, as defined by Lada (1987), using all four IRAC fluxes. The spectral index $\alpha_{\text {IRAC }}$ was determined from the slope of the SED measured between 3.6-8.0 $\mu \mathrm{m}$ (see Lada et al. 2006). The flux error measurements were used as the quadratic weight when performing the least-squares fits. YSO candidates with $\alpha_{\text {IRAC }}>-0.3$ were classified as Class I and $-0.3>\alpha_{\text {IRAC }}>-1.6$ as Class II. Since Class III candidates $\left(-1.6>\alpha_{\text {IRAC }}>-2.56\right)$ are the most affected by biases, they were discarded from our analysis. The main reason for this exclusion is the high level of contamination of asymptotic giant branch (AGB) stars throughout the Galactic plane that harbor thin disks and mimic the SED of genuine Class III YSOs (Robitaille et al. 2008). Furthermore, carbon-rich red giant envelopes can also produce PAH emission at $8 \mu \mathrm{m}$ and therefore artificially increase the number of Class III candidates (e.g., Strafella et al. 2015). We note that this classification scheme is based on what Greene et al. (1994) proposed, with the exception 


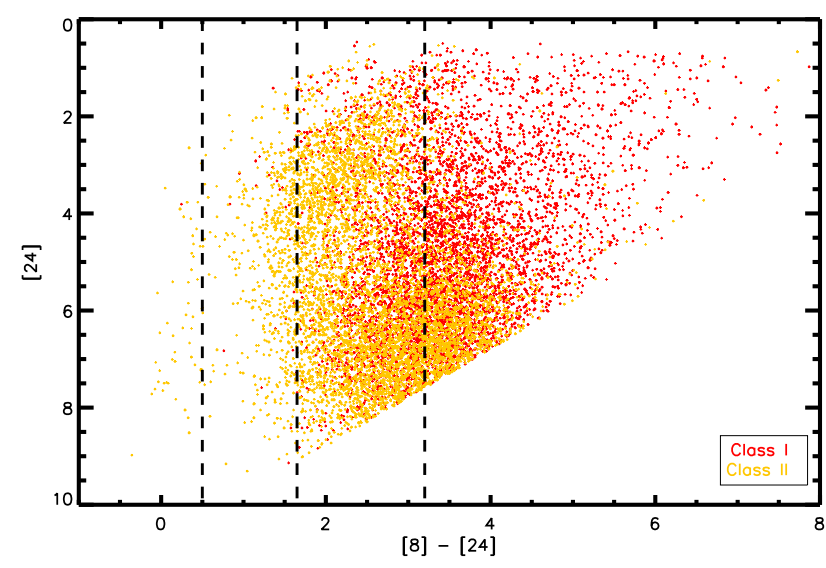

Fig. 2. YSO candidates classified using the IRAC fluxes in a [24] versus [8]-[24] diagram. The vertical dashed lines indicate the expected regions for the different evolutionary stages, based on the [8]-[24] colors, where Class I sources should have [8]-[24] > 3.2, Class II sources $1.6 \leq[8]-[24] \leq 3.2$, Class III sources $0.5 \leq[8]-[24] \leq 1.6$, and mainsequence stars should have $[8]-[24]<0.5$.

that we do not distinguish between "flat spectrum" and Class I types, but consider both as Class I. This led to 13968 YSOs that were classified as Class I and 28317 as Class II.

However, the color criterion was developed by Gutermuth et al. by using deep Spitzer/IRAC imaging of nearby starforming clouds, and studies have shown that significant false positives were selected when this criterion was applied to GLIMPSE data of more distant star-forming regions (e.g., Povich et al. 2009, 2013). Therefore, to further ensure a more robust sample of Class I and Class II candidates, we also identified the different classes using IRAC [3.6]-[4.5]/[5.8]-[8.0] color-color diagrams (e.g., Allen et al. 2004). The IRAC colorcolor diagram with the YSO candidates and respective evolutionary classifications is displayed in Fig. 1. We find that 10694 ( 77\%) Class I and $18209(\sim 64 \%)$ Class II sources that are classified based on their spectral indices have the same evolutionary stage according to their position in the color-color diagram. This YSO sample is considered in our further analysis.

Another concern arising from our YSO classification is the effect of extinction along the line of sight, which is especially problematic in the Galactic plane region. This effect may compromise some of the measured IRAC fluxes and therefore affect our YSO classification, which is inferred from the spectral indices. The reddening effect on sources that is due to extinction can potentially bias our YSO sample toward the detection of false-positive YSOs and also move evolved YSO toward earlier evolutionary stages. In order to evaluate the effect caused by extinction, we performed an independent source classification using [8] and [24] for sources with a $24 \mu \mathrm{m}$ counterpart in the MIPSGAL catalog, since extinction is lower between 8 and $24 \mu \mathrm{m}$. In Fig. 2 we display the 12997 YSO candidates that have a $24 \mu \mathrm{m}$ counterpart in a [8] vs. [24] diagram. By relating the SED spectral indices with the [8]-[24] color, we reclassified our sources and compared the results with the $\alpha_{\text {IRAC }}$ classification.

The results of this comparison, summarized in Table 1, show that the majority $(\sim 64 \%)$ of the sources classified with the four IRAC bands maintain the same classification when reclassified using the [8]-[24] colors. Although there is some uncertainty between the classes, it is reassuring to know that only a small fraction of YSOs $(\sim 0.4 \%)$ are reclassified as pre-main-sequence stars in the [8]-[24] color diagram $([8]-[24]<0.5)$.
Table 1. Results of the comparison between the classification using $\alpha_{\text {IRAC }}$ and [8]-[24] criteria for YSO candidates with $24 \mu \mathrm{m}$ detection.

\begin{tabular}{|c|c|c|}
\hline${ }_{[8]-[24]^{(\mathrm{b})}} \alpha_{\text {IRAC }}+$ color $^{(a)}$ & Class I (6157) & Class II $(6840)$ \\
\hline Class I & $3705(60.2 \%)$ & $1702(24.9 \%)$ \\
\hline Class II & $2423(39.4 \%)$ & $4543(66.4 \%)$ \\
\hline Class III & $28(0.4 \%)$ & $538(7.9 \%)$ \\
\hline Star & $1(0.0 \%)$ & $57(0.8 \%)$ \\
\hline
\end{tabular}

Notes. (a) YSO candidates with $24 \mu \mathrm{m}$ detection classified by $\alpha_{\text {IRAC }}$ criteria and color-color IRAC diagram. (b) YSO candidates classified using [8]-[24] colors.

However, $24 \mu \mathrm{m}$ emission is usually diffuse or even saturated in the inner regions of the bubbles because of the emission of small dust grains inside the ionized region of the bubble and the PDR. Therefore, only 28\% (1126 out of 4044) of the YSO candidates located inside a bubble have a $24 \mu \mathrm{m}$ counterpart, while outside the bubbles we find $48 \%$ (11 871 out of 24859 ). Nevertheless, more than $99 \%$ of the Class II and Class I YSO candidates with a $24 \mu \mathrm{m}$ counterpart that were classified using $\alpha_{\text {IRAC }}$ are still classified as a YSO after the [8]-[24] classification, which supports our assumption that the $\alpha_{\text {IRAC }}$ classified sample is not significantly contaminated by stellar objects.

\subsection{Dust temperature and column density distribution in the vicinity of $\mathrm{H} / \mathrm{l}$ regions}

The four intensity maps from $160 \mu \mathrm{m}$ to $500 \mu \mathrm{m}$ were used to compute the column density and the dust temperature $\left(T_{\mathrm{d}}\right)$ maps. First the three intensity maps at $\lambda<500 \mu \mathrm{m}$ were convolved with a Gaussian to degrade their spatial resolution: after this step, all the maps have an equivalent resolution of $36^{\prime \prime}$ of the $500 \mu \mathrm{m}$ (PLW) band; then the three maps were regridded onto the same spatial grid of the PLW image. After this step, the four maps can be stacked and $N\left(\mathrm{H}_{2}\right)$ and $T_{\mathrm{d}}$ can be derived by fitting a modified blackbody pixel by pixel: it is assumed that the dust emission can be modeled as $I_{v}=\kappa_{v} \Sigma B_{v}\left(T_{\mathrm{d}}\right)$, where $B_{v}\left(T_{\mathrm{d}}\right)$ is the Planck function at temperature $T_{\mathrm{d}}, \Sigma=\mu_{\mathrm{H}_{2}} m_{\mathrm{H}} N\left(\mathrm{H}_{2}\right)$ is the gas surfacedensity distribution with $\mu_{\mathrm{H}_{2}}=2.8$ (molecular weight), $m_{\mathrm{H}}$ is the mass of the hydrogen atom, and $N\left(\mathrm{H}_{2}\right)$ is the column density of molecular hydrogen. The dust opacity per unit mass (assuming a gas-to-dust ratio of 100) is expressed as a power law: $\kappa_{v}=0.1 \times(300 \mu \mathrm{m} / \lambda)^{2} \mathrm{~cm}^{2} / \mathrm{g}$. The assumption on the molecular weight and on the functional form of $\kappa_{v}$ are typical of the Hi-GAL program (e.g., Elia et al. 2013).

The fitting procedure was executed with a $\mathrm{C}$ code that takes the four images as input. The code creates a grid of models, as in Pezzuto et al. (2012), according to the needs of the user: since in our case the dust emissivity index $\beta$ is fixed to 2 , the grid is built by varying only the temperature in the range $5 \leq T_{\mathrm{d}}(\mathrm{K}) \leq 50$ in steps of $0.01 \mathrm{~K}$. For each temperature, the grid gives the intensity at the four wavelengths corresponding to a column density of $1 \mathrm{~g} / \mathrm{cm}^{2}$, and $I_{v}$ is linear in $N\left(\mathrm{H}_{2}\right)$ so that the column density for a given SED $f_{i}$ can be computed with a straightforward application of the least-squares technique. Then, for each pixel we have a set of pairs $\left(T_{\mathrm{d}_{i}}, N\left(\mathrm{H}_{2}\right)\right)$, with $j$ running over all the models in the grid; the pair with the lowest residuals is kept as best-fit model for that pixel. Color corrections were not computed. The uncertainty associated to $I_{v}$ was assumed to be $20 \%$ at all wavelengths. More details on the production of the column density and temperature maps can be found elsewhere (Pezzuto et al., in prep.; Schisano et al., in prep.). 

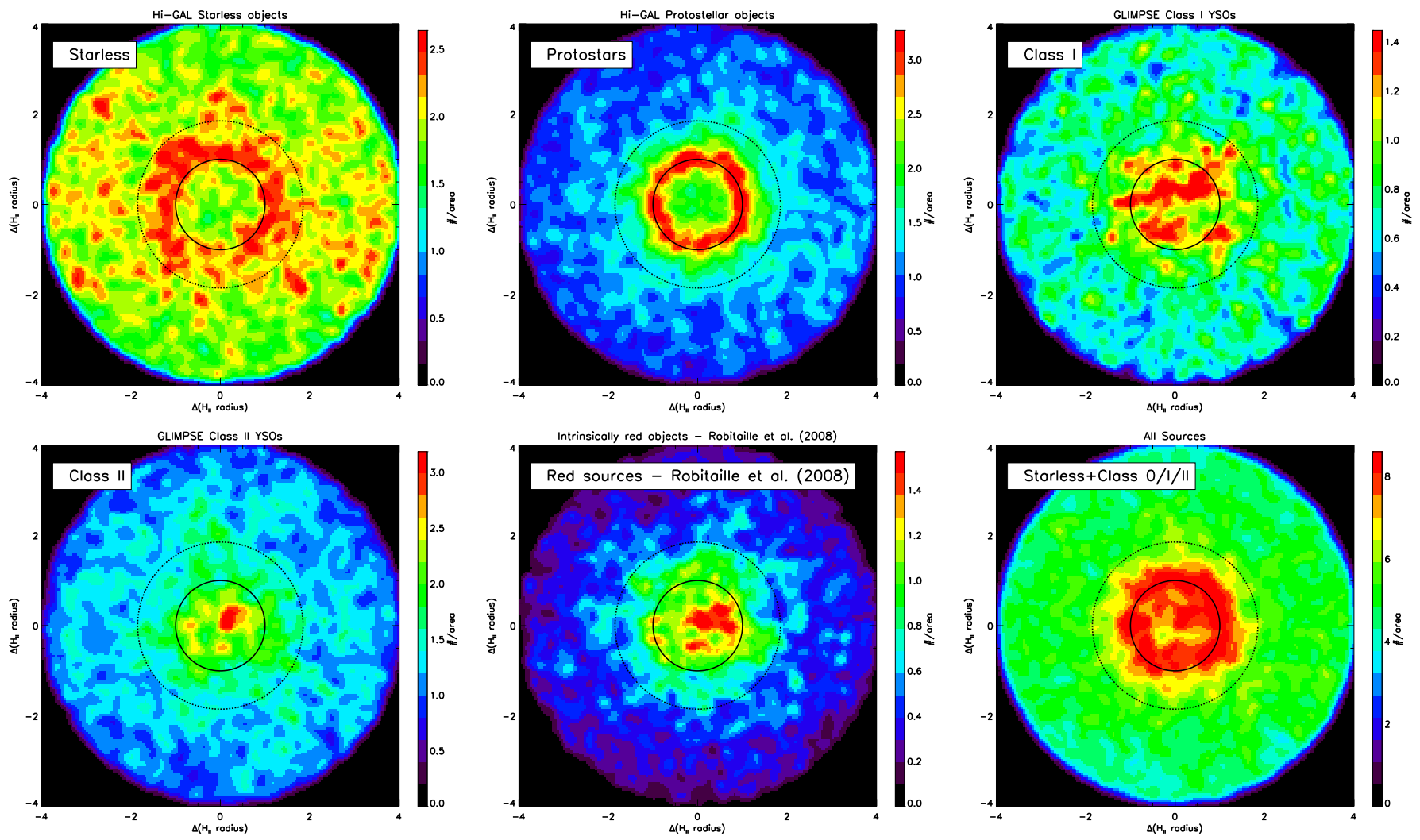

Fig. 3. Surface density maps for all star-forming objects - Hi-GAL clumps, IRAC YSOs, and intrinsically red sources from Robitaille et al. (2008) - associated with the bubble sample. The spatial scales of the maps are normalized by the bubble radius (solid black circle). The dashed black circle represents the average shell radius $\left(R_{\text {shell }}=1.85 \times R_{\text {eff }}\right)$. The maps are smoothed by convolving with a Gaussian with a size of $F W H M=0.25 R_{H_{I I}}$.

This method was produced to increase the computational speed needed in particular for the Herschel Hi-GAL fields that were converted into larger mosaics. Comparison with previous Hi-GAL column density maps following a different approach (e.g., Elia et al. 2013; Schisano et al. 2014) reveals negligible inaccuracies between these two methods.

\section{Results}

\subsection{Distribution of star-forming sources related to ionized regions}

The spatial information of all the associated star-forming objects was compiled into surface density maps. To take the different sizes of the bubbles into account, we normalized the $l$ and $b$ offsets from the center of the bubbles by the respective $R_{\text {eff }}$ of the bubbles. A grid of 80 by 80 pixels with a pixel size of $0.05 R_{\text {eff }}$ was generated following the orientation of the Galactic coordinates, with the $x$-axis aligned with the direction of the Galactic latitude $l$ and the $y$-axis with the Galactic longitude $b$. Then we measured the number counts for each star-forming object type at each pixel. As a final step, the maps were smoothed by convolving them with a Gaussian with $F W H M=0.25 R_{\text {eff. }}$ In Fig. 3 we show the surface density maps for each star-forming object type.

We note that radial velocity measurements of YSOs and clumps that are spatially associated with bubbles have been found to be consistent with ionized gas of H II regions (e.g., Martins et al. 2010; Hou \& Gao 2014; Deharveng et al. 2015; Liu et al. 2015, 2016). We therefore assumed that star-forming objects that are found within the edges of bubbles are physically associated with them.

The surface density maps provide a clear picture of where star-forming objects are typically located in the vicinity of bubbles. These maps can be interpreted as probability function maps, revealing where star-forming objects at a given evolutionary stage are most probably located relative to a bubble.

Another visualization of the overdensity of star-forming objects is expressed as azimuthally averaged profiles of the surface density map, as displayed in Fig. 4. The surface density profile of all sources shows two significant peaks, one toward the center and the other toward the rim of the bubbles. The individual profiles show that Class II YSOs dominate the inner regions of the bubbles, while younger starless and protostellar objects are mostly seen toward the edges. In particular, the peak of each individual profile follows a gradient in age.

In order to analyze how significant the surface density of star-forming sources is "inside" the bubble and "outside" the bubbles, we first need to define the radius of the shells. In previous studies the area up to 1.6 and 2 times the $R_{\text {eff }}$ of the bubble was considered as part of the bubble, as the surface density of star-forming objects decreased to the level of the background (e.g., Kendrew et al. 2012; Thompson et al. 2012). Here, however, we opted to take the different thicknesses of the bubbles into account. Therefore, we defined the radius of the shell $\left(R_{\text {shell }}\right)$ of the bubble as the sum of the angular thickness of the bubbles (provided from the MWP catalog) with $R_{\text {eff }}$. On average, $R_{\text {shell }}$ of our sample is $\sim 1.85 \pm 0.21 \times R_{\text {eff }}$, which is approximately the same as the constant inner radius assumed by 


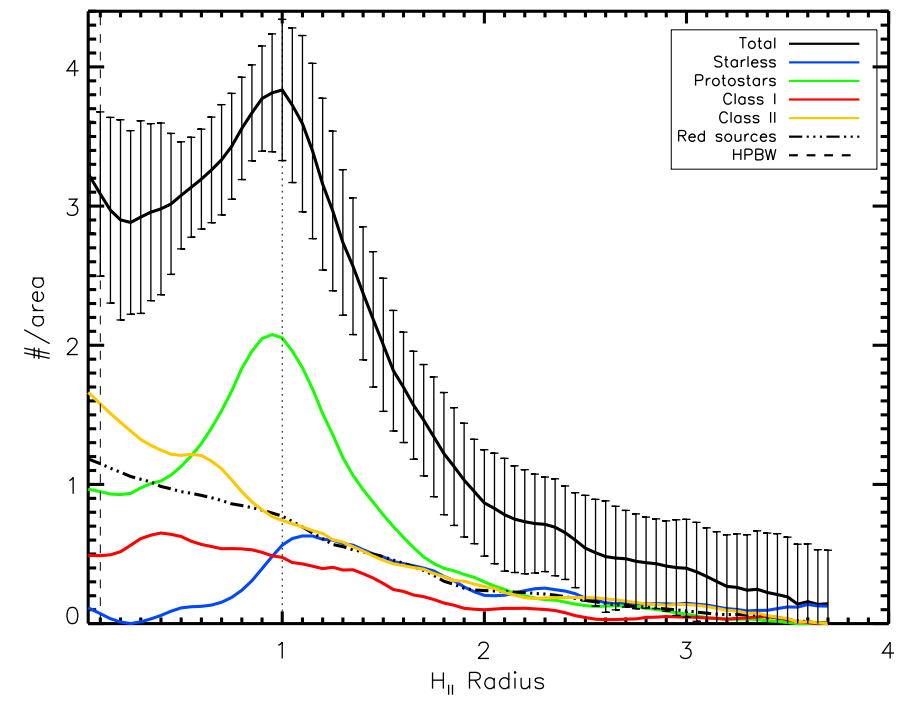

Fig. 4. Mean radial source density profiles as a function of distance to the central position of the H II regions, obtained by azimuthally averaging from the surface density maps. The black solid line represents the cumulative count of the different source types (color-coded solid lines). The black dash-dotted line represents the mean radial source density profile of the intrinsically red sources from Robitaille et al. (2008). The distance is normalized by the radius of the H II regions (dotted vertical line). The error bars show the $( \pm 1 \sigma)$ dispersion of the radial profiles. The dashed vertical line represents the HPBW $\left(=0.125 R_{\text {eff }}\right)$ of the smoothing gaussian used in the surface density maps.

previous authors. Considering the surface density of all starforming sources (excluding Class III) inside the shell $\left(<R_{\text {shell }}\right)$ and outside $\left(R_{\text {shell }}<r<4 R_{\text {eff }}\right)$, we estimate that the weighted mean ratio is $1.8 \pm 0.7$, weighted by the total number of sources ( $\left.<4 R_{\text {eff }}\right)$ per bubble. This means that we typically find $80 \%$ more star-forming objects per unit area toward the direction of bubbles than outside. Nevertheless, we note that some overlap between other bubbles and the considered outer area $\left(R_{\text {shell }}<r<4 R_{\text {eff }}\right)$ may contaminate our estimates of the surface density in these regions, especially for smaller bubbles, which are not considered here. Although we expect this effect to be minor due to the large statistics, this may slightly increase the number of sources located outside the bubbles. Therefore we take the $\sim 80 \%$ ratio as a lower estimate.

However, since the selection criteria used for the YSO candidates enforces high-quality detection (magnitude errors $<0.2 \mathrm{mag}$ ) in all four IRAC bands, a lack of detection of YSO candidates at the rim of the bubbles may be due to the very strong diffuse emission from the PDR at $8 \mu \mathrm{m}$. To ensure that the overdensity of YSO sources in the inner part of the bubbles is not biased for this reason, we dropped the requirement of having quality photometric measurements at [8.0] band and repeated our analysis by applying the Gutermuth et al. color criteria to identify YSOs. We also classified them into Class I and II using the spectral index slope and the color-color diagram, as in Sect 3.2. To compensate for the poor quality of the photometric flux at $8.0 \mu \mathrm{m}$, we made use of the $24 \mu \mathrm{m}$ band measurement to evaluate the spectral index of the YSO candidates whenever available.

By applying these criteria, we obtained sample of 11330 Class I and 27879 Class II YSO candidates, which represents an increase of $6 \%$ and $53 \%$, respectively, when compared with our previous criteria. In this sample we found that 6125 $(\sim 54 \%)$ Class I and $7448(\sim 27 \%)$ Class II candidates have a measured MIPS [24] counterpart. The resulting spatial distribution of sources, displayed in Fig. A.1, shows almost exactly the same spatial YSO distribution as we obtained with our previous criteria to construct Fig. 3. The increase in the number of YSO candidates further enhances the statistics of YSOs inside bubbles. This indicates that the overdensity of Class I and Class II YSOs in the inner part of the bubbles are not an artefact that is due to the possibility of fewer detections caused by the PDR at the rim of the bubbles.

Last, to certify the reliability of the obtained result, we also analyzed in the same manner a more recent catalog of $~ 133980$ Class I/II YSOs candidates identified from the ALLWISE catalog (Cutri \& et al. 2013) based on a vector machine selection (Marton et al. 2016). We find that 46761 of the sources lie in the same area as is covered by the Hi-GAL survey of the inner Galactic plane. The WISE photometric data were combined with reliable 2MASS photometric measurements and Planck dust opacity values. We found $24517(\sim 52.4 \%)$ sources within the $<4 \times R_{\text {eff }}$ criterion and 5045 sources are located inside the bubble radii $\left(<R_{\text {eff }}\right)$. In Fig. A.2 we show the comparison of the surface density maps of the combined Class I and II YSO candidates classified here in Sect. 3.2 using IRAC fluxes and the Class I/II candidates taken from the WISE catalog. The YSO candidates of both selections peak at the center of the bubble with a surface unit of $\sim 4-5$ per bubble area $\left(=\pi R_{\text {eff }}^{2}\right)$. The main difference is mostly in the background sources with a lower value and in the flatter distribution of WISE YSO candidates. That different observation sets and YSO classifications provide the same conclusion considerably strengthens this result.

\subsection{Dynamic age estimates of $H$ II regions}

The analytical solutions proposed by Spitzer (1978) and Dyson \& Williams (1980) are commonly used to derive an age estimate by determining the required expansion time for an H II region to reach its current size (e.g., Zavagno et al. 2007). However, the predictions used in their method neglect the effect of the surrounding turbulent pressure, which can influence the size of the H II region, as demonstrated in Tremblin et al. (2012). Analytical solutions and numerical simulations performed in Tremblin et al. (2014) demonstrated that the expansion of H II regions is slowed down by turbulent ram pressure $\left(P_{\text {turb }}\right)$ of the environment until it reaches quasi-static equilibrium with the pressure of the ionized gas $\left(P_{\mathrm{II}}\right)$. These results allowed Tremblin et al. to develop a reliable method to determine the dynamic ages of $\mathrm{H}$ II regions. With the use of radio continuum flux measurements and by applying the Larson laws (see Larson 1981) to infer $P_{\mathrm{II}}$ and $P_{\text {turb }}$, respectively, dynamic age estimates were obtained by comparing the results with the isochrones provided by the grid of 1D models of expanding $\mathrm{H}$ II regions. The derived dynamic ages agree well with the photometric ages of the ionizing stars in well-known regions (e.g., Rosette, RCW 36, RCW 79, and M16, Tremblin et al. 2014). We found that 182 Galactic bubbles from our sample have a determined distance and associated radio flux measurements and are therefore eligible for determining a dynamic age using this method. For this purpose, we made use of the $1.4 \mathrm{GHz}$ radio continuum flux measurements performed on the NVSS images, cataloged in Condon et al. (1998), for the northern part of the Galactic plane (120 found), and the Parkes-MIT-NRAO (PMN) survey at $4.85 \mathrm{GHz}$ (Wright et al. 1994) for the southern part (62 found). The distances of the Galactic bubbles were obtained from the WISE catalog of Galactic H II regions (Anderson et al. 2014). 


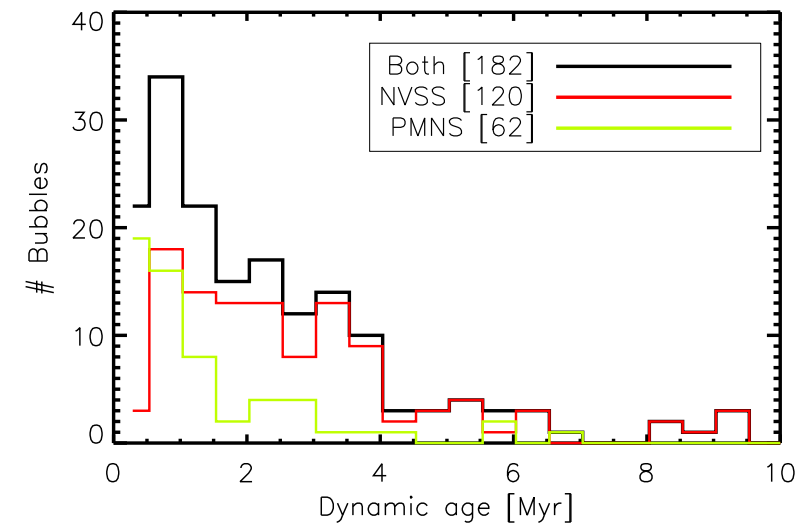

Fig. 5. Dynamic age histogram for a subsample of 182 bubbles estimated using the NVSS (in red) and PMNS (in green) surveys.

Following Tremblin et al. (2014), we first calculated the rms electron density $\left\langle n_{\mathrm{II}}\right\rangle$ (see Martín-Hernández et al. 2005):

$\left\langle n_{\mathrm{II}}\right\rangle=\frac{4.092 \times 10^{5} \mathrm{~cm}^{-3}}{\sqrt{b\left(v, T_{\mathrm{e}}\right)}}\left(\frac{S_{v}}{\mathrm{Jy}}\right)^{0.5}\left(\frac{T_{\mathrm{e}}}{10^{4} \mathrm{~K}}\right)^{0.25}\left(\frac{D}{\mathrm{kpc}}\right)^{-0.5}\left(\frac{D_{\mathrm{eff}}}{\prime \prime}\right)^{-1.5}(1)$

$b\left(v, T_{\mathrm{e}}\right)=1+0.3195 \log \left(\frac{T_{\mathrm{e}}}{10^{4} \mathrm{~K}}\right)-0.2130 \log \left(\frac{v}{\mathrm{GHz}}\right)$,

where $S_{v}$ is the radio continuum integrated flux at frequency $v$ taken from the NVSS and PMN catalogs, $D_{\text {eff }}$ is the angular diameter of the bubble, and $D$ the distance from the Sun. $T_{e}$ is the electron temperature in the ionized plasma, and it was derived by using the linear relation with galactocentric distance obtained by Balser et al. (2011). Then, the ionized gas pressure can be derived as follows:

$P_{\mathrm{II}}=2\left\langle n_{\mathrm{II}}\right\rangle k_{\mathrm{b}} T_{\mathrm{e}}$.

Finally, we derive an estimate of the dynamic age for our subsample of 182 bubbles by comparing it with the isochrones of the 1D simulations performed in Tremblin et al. (2014). The distribution of the resulting ages is presented in Fig. 5. We find a peak around $0.75 \mathrm{Myr}$ in the histogram, and the large majority of the $\mathrm{H}$ II regions have ages younger than $4 \mathrm{Myr}(\sim 80 \%)$. Similarly, Tremblin et al. also found that most of the derived dynamic ages for a sample of 119 regions that were detected using radio continuum measurements obtained by the Green Bank Telescope at $9 \mathrm{GHz}$ (Anderson et al. 2011) as part of the H II Region Discovery Survey (HRDS) ${ }^{6}$ are younger than 4 Myr. This distribution can be related with the typical lifetime of high-mass stars. A main-sequence spectral type O5 star, for example, has an expected lifetime of 4 Myr (Allen 1973).

We note that the minimum angular size criteria of $R_{\text {eff }}>72^{\prime \prime}$ imposed for the MWP bubbles biases ages toward older bubbles. As an extreme example, a bubble with an angular radius of $72^{\prime \prime}$ located at a far heliocentric distance of $15 \mathrm{kpc}$ would correspond to a physical radius size of $\sim 5.2 \mathrm{pc}$. For the Tremblin et al. models, this corresponds to the expected size of a bubble created by an $\mathrm{O} 5$ star expanding into a $3500 \mathrm{~cm}^{-3}$ uniform medium during 2 Myr. This means that we might be excluding bubbles at earlier stages of expansion (smaller radius) that are located at greater distance. However, this criterion does not exclude the potential detection of older bubbles. This age distribution may well reflect the typical lifetime of IR-bright $\mathrm{H}$ II regions located in the

\footnotetext{
6 http://www.cv.nrao.edu/hrds
}

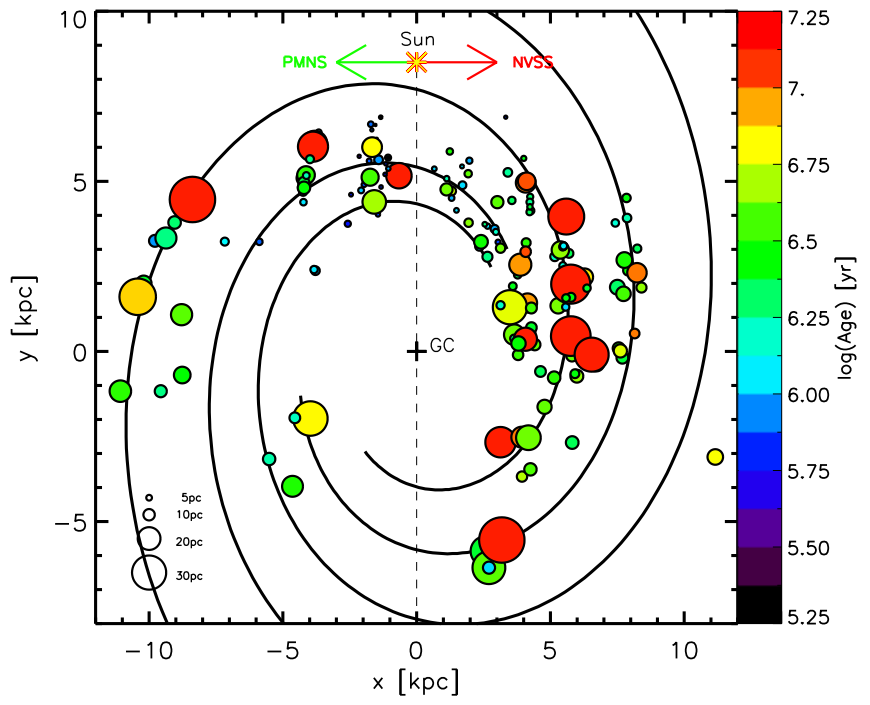

Fig. 6. Bubble distribution in Galactocentric coordinates with their respective diameter and age. The black solid curves represent the position of the four Galactic spiral arms based on Russeil (2003).

Milky Way to be around $<4$ Myr, for the majority of bubbles, or that IR-bright $\mathrm{H}$ II regions are simply more visible during the earlier stages of expansion.

In Fig. 6 we present a top view of the location of the 182 bubbles in the Galactic plane, with their respective dynamic ages and sizes. In addition to finding more bubbles with an NVSS flux measurement in the northern Galactic plane (120) than PMNS flux measurements in the southern Galactic plane (62), we also find bubbles at larger distances in the northern part. The main reason for this is the lower detection limit of the NVSS survey ( 2.5 mJy) compared with the PMNS survey ( 20-40 mJy). Furthermore, the bubbles tend to be located in the Galactic spiral arms taken from Russeil (2003).

\subsection{IR emission from bubbles and shell density estimates}

The multiwavelength images available throughout the Galactic plane, in particular the Hi-GAL, GLIMPSE and MIPSGAL surveys, allow a thorough analysis of the local environment surrounding our sample of bubbles.

Averaged column density and $70 \mu \mathrm{m}$ and $24 \mu \mathrm{m}$ maps were generated in order to obtain an overview of the typical bubble environment. For each bubble we considered the squared area centered on the bubble of a length of $8 \times R_{\text {eff }}$, normalized by its respective central value. The averaged maps were then generated by regridding all the maps to the same pixel size of $0.05 \times R_{\mathrm{eff}}$ and were averaged on a pixel-by-pixel basis for all the bubbles. The resulting averaged maps and their respective azimuthally averaged profiles are displayed in Fig. 7. We note that the averaged bubbles are well resolved at the lowest resolution of the column density maps (36.3") and therefore no convolution was considered when performing the averaged maps.

As expected, most of the $24 \mu \mathrm{m}$ emission that traces the ionized gas is detected in the inner part of the bubbles, while the $70 \mu \mathrm{m}$ emission, which traces the ionized emission and the PDR, clearly peaks at the edges of the bubbles $\left(R_{\text {eff }}\right)$. We also find that the column density is lower in the inner part of the bubbles and increases at the edge of the bubbles, which is probably the effect of material that is swept up by the expansion of the H II region. 

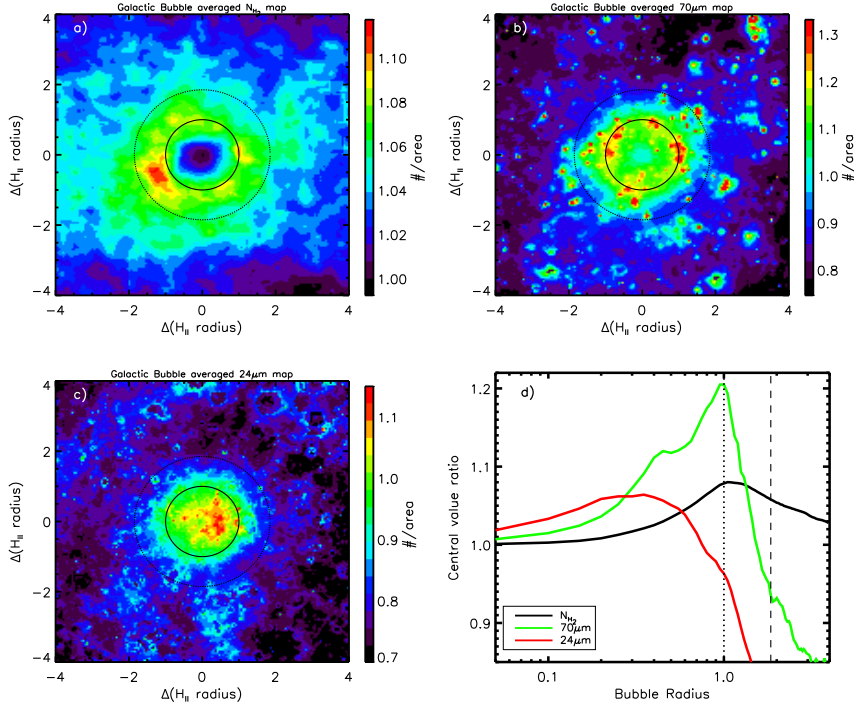

Fig. 7. Averaged column density and $70 \mu \mathrm{m}$ and $24 \mu \mathrm{m}$ maps of the Galactic bubble sample, panels $a, b$, and $c$, respectively. The map units are normalized by the value in the central position of the bubbles. $R_{\mathrm{eff}}$ and $R_{\text {shell }}$ are represented by the solid and dashed black circle, respectively. $d$ ) Circularly averaged profiles of the averaged column density (black solid), $70 \mu \mathrm{m}$ (green solid), and $24 \mu \mathrm{m}$ (red solid) maps. The vertical dotted and dashed lines indicate $R_{\text {eff }}$ and $R_{\text {shell }}$, respectively.

Furthermore, Herschel column density maps and bubble physical sizes allow us to estimate the mass of the bubble $\left(M_{\text {bub }}\right)$. Using the 182 bubble sample from Sect. 4.2 for which we have kinematic distances, we estimated the background for each bubble by taking the minimum value around a $8 \times 8 R_{\text {eff }}$ box centered at each bubble. A median filter of $35^{\prime \prime}$ size was applied before the background subtraction to avoid poorly SED fitted pixels of the column density maps. After subtracting the local background (minimum value in the median filtered map), we calculated the mass for each bubble contained inside the shell of the bubble. We computed $M_{\text {bub }}$ by summing all the pixels inside $R_{\text {shell }}$ of the bubble, as follows:

$M_{\text {bub }}=\sum N_{\mathrm{H}_{2}}\left(r<R_{\text {shell }}\right) \times\left(\frac{A_{\text {pixel }}}{\mathrm{cm}^{2}}\right) \times \mu_{\mathrm{H}_{2}} \times m_{\mathrm{H}}(\mathrm{g})$,

where $A_{\text {pixel }}$ is the pixel area in $\mathrm{cm}^{2}, m_{\mathrm{H}}$ is the atomic molecular of hydrogen, and $\mu_{\mathrm{H}_{2}}$ is the mean molecular weight per $\mathrm{H}_{2}$ molecule (cf. Kauffmann et al. 2008).

Provided with mass estimates, we then calculated the mean volume density of the rim $\left(\rho_{\text {shell }}\right)$ by assuming that the bubbles have a spherical geometry and that all the $M_{\text {bub }}$ is confined between $R_{\text {eff }}$ and $R_{\text {shell }}$, with no contribution from the inner part of the bubble. In other words, we considered that $M_{\text {bub }}$ was contained in the volume of the shell $\left(V_{\text {shell }}\right)$ :

$V_{\text {shell }}=\frac{4}{3} \pi\left(R_{\text {shell }}^{3}-R_{\text {eff }}^{3}\right)\left(\mathrm{cm}^{-3}\right)$.

Finally, the $\rho_{\text {shell }}$ was calculated by considering that the entire $M_{\text {bub }}$ is contained inside the $V_{\text {shell. }}$. In Fig. 8, we express our density estimates in the form of the average number density $\left\langle n_{\text {shell }}\right\rangle$ as a function of the dynamic age for 182 bubbles. This plot shows a weak but significant trend between the mean density at the shell of the bubbles and their dynamic ages. We find that typically, molecular gas concealed in the shells of the bubbles has a higher density at earlier stages of the bubble expansion, which could therefore suggest that bubbles are probably formed

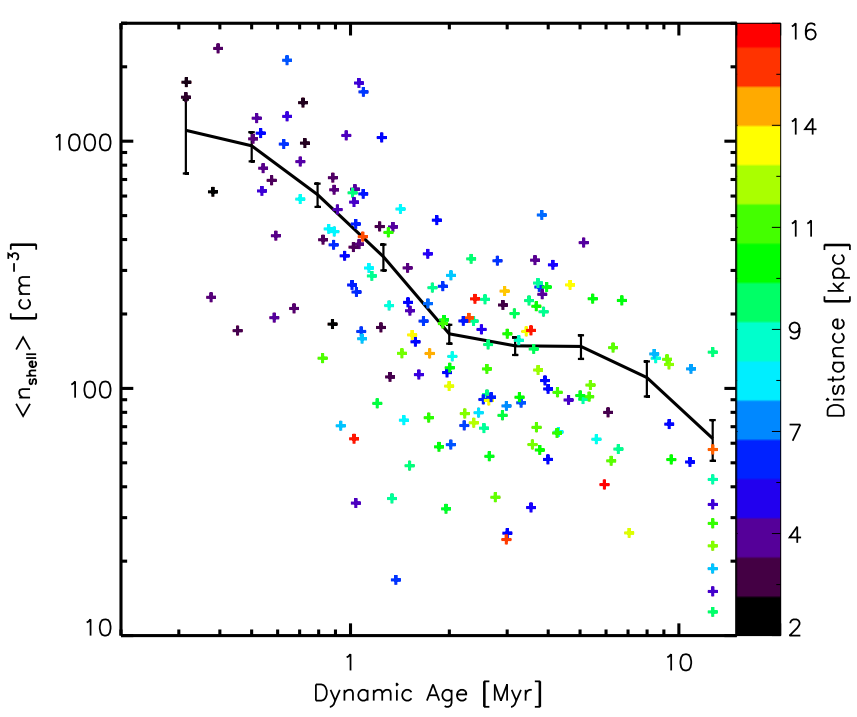

Fig. 8. Average number density inside the shell of the bubbles as a function of dynamic age of the bubbles. The values are color-coded with the distance of their respective bubble. The solid line represents the average value, with the respective error bars representing the standard deviation of the mean. The molecular cold gas located inside the shell ( $\left.R_{\text {eff }}<r<R_{\text {shell }}\right)$ of the bubbles tends to be denser for younger bubbles.

in high-density regions. The decreasing trend would result from the increasing volume of the shell as the bubble expands and presumably converts part of its mass into stars.

\section{Discussion}

\subsection{Star formation evolutionary gradient and timescales}

The comprehensive statistical compilation of the spatial location of star-forming objects at different evolutionary stages, performed in Sect. 4.1, reveals a clear evolutionary sequence. Thompson et al. (2012) indicated this possibility when they found that intrinsically red sources (more evolved sources) from Robitaille et al. (2008) had a flat distribution inside the bubbles (see Figs. 3 and 4), while younger sources from the Red MSX Source (RMS) catalog (Urquhart et al. 2008) were found to peak at the edges of the bubbles.

Interestingly, Class II YSOs in the inner part of the bubbles are found for bubbles that have younger dynamic ages than the typical lifetime of low- and intermediate-mass Class II objects $\sim 2 \pm 1 \mathrm{Myr}$ (Evans et al. 2009). This suggests that these YSOs have probably undergone their formation process before the expansion of the bubble, possibly as part of the same star-forming complex that gave birth to the ionizing massive stars that are responsible for the expansion of the bubbles.

In cluster-forming environments, massive stars are expected to form after low-mass stars have completed their accretion phase (Kumar et al. 2006). Moreover, the well-studied case of the NGC 6357 complex, which is included in our sample of 1360 bubbles, is a very good representative of this timescale issue. Its relative proximity $\sim 1.7 \mathrm{kpc}$ (Russeil et al. 2010) allowed us to detect several Class II YSO ( 220) candidates in deep IRAC observations around this complex, which are found spatially distributed around the $\sim 1$ Myr OB cluster that is ionizing the H II region (Fang et al. 2012). Using less sensitive IRAC images, we find 64 Class II YSOs located in the inner part of the bubble $\left(<R_{\text {eff }}\right)$, with a surface density $\sim 5.3$ times higher than the outer region in its proximity $\left(R_{\mathrm{eff}}<r<4 R_{\mathrm{eff}}\right)$. The location 
of the Class II YSOs and the estimated age of the cluster indeed suggest that these YSO were probably formed as part of the OB cluster that ionizes the region.

Moreover, a high-density environment of cold molecular gas is required for massive star-formation to occur, which is a prerequisite for the formation of H II regions. This can be easily related with the high-density shells of molecular gas found for bubbles at earlier stages of the bubble expansion (see Fig. 8). Given that massive star formation is expected to be faster than low-mass star formation (McKee \& Ostriker 2007; Zinnecker \& Yorke 2007), it is probable that a few remaining sources were already undergoing formation at the moment the dense medium of the "parent" cloud started to become ionized. Furthermore, radiative magnetohydrodynamic simulations of expanding $\mathrm{H}$ II regions show that denser clumps are more resistant to the expansion (Geen et al. 2015). Although we did not find significant evidence for the existence of dense clumps inside the bubbles, it is possible that photoevaporation by UV photons of the massive stars partially dissipated their envelope because of their proximity (Gritschneder \& Burkert 2014).

However, given the generally large distances of the bubbles and the sensitivity of the GLIMPSE data, most of the YSOs are probably intermediate- or high-mass objects and are therefore expected to have lifetimes shorter than 2 Myr. Therefore we also have to consider the possibility that the YSOs seen inside the bubble could have been formed through triggering by the bubble. An interesting alternative is proposed in Gritschneder \& Burkert (2014), who presented a model of the dynamics of H II regions and triggered stars. The authors predict that triggered stars that formed inside the shell of the bubbles may migrate into the interior of $\mathrm{H}$ II regions if the expansion occurs under particular conditions, depending on the initial density of the environment, the formation time of the triggered star, and the flux of the ionizing star(s). For cases such as Orion, the authors estimate that triggered stars may migrate back to the center of the $\mathrm{H}$ II region within $0.6 \mathrm{Myr}$. More importantly, their models predict that more evolved objects are seen closer to the ionizing star(s), following an age gradient.

Altogether, these arguments provide plausible scenarios for the observed high concentration of YSOs in the interior of the bubbles and the evolutionary trend in the surface density maps.

Nevertheless, accurate measurements of proper motions of YSO sources is crucial to understand this evolutionary sequence toward the interior of bubbles for Class II sources. When we can determine whether the YSOs are indeed migrating toward the center of H II regions, we will be able to provide a clear picture of how feedback interacts with the star-forming sources and gain insight into the initial conditions of $\mathrm{H}$ II regions.

For this reason, we would like to point out that high-precision infrared astronomic instruments, such as the Gemini Multi-conjugate Adaptive Optics System (GeMS; Rigaut et al. 2014), can reach precisions on the order of $\sim 150 \mu$ as (Lu et al. 2014) and could be used in the future to address this issue. Furthermore, the instrument has previously been successfully used to study the RCW $41 \mathrm{H}$ II region (Neichel et al. 2015). In order to estimate the feasibility of such a measurement, we take the expected velocity in the plane of the sky of $\sim 2 \mathrm{~km} \mathrm{~s}^{-1}$ for a migrating YSO close to the center of an $\mathrm{H}$ II region from the model of Gritschneder \& Burkert, assuming an averaged inclination projection angle of $45^{\circ}$. For such a case we would expect for a $\mathrm{H}$ II regions located at $\sim 2 \mathrm{kpc}$ distance a proper motion velocity of $\sim 210 \mu \mathrm{as} / \mathrm{yr}$. This is extremely encouraging for future proposals that would allow probing the internal dynamic motions of star-forming objects inside bubbles.
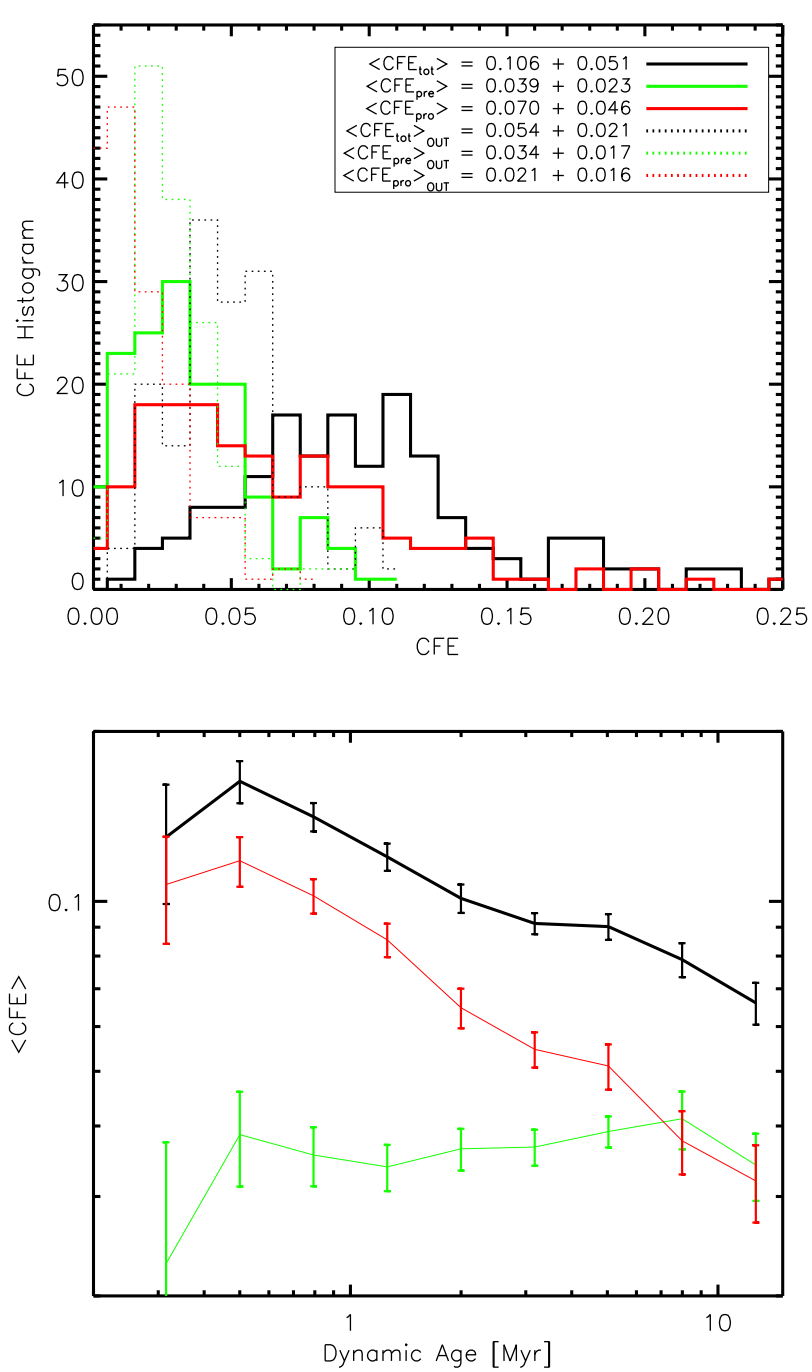

Fig. 9. Top: Histogram of the distribution of the total CFE (solid black), prestellar (solid green), and protostellar (solid red) sources inside the bubbles $\left(<R_{\text {shell }}\right)$ and outside $\left(R_{\text {shell }}<r<4 \times R_{\text {eff }}\right)$ (dotted). Bottom: CFE as a function of the dynamic age of the bubbles.

\subsection{Clump formation efficiency of bubbles}

A crucial aspect of the overall impact of H II regions on the star formation process is to understand how efficient the conversion of the neutral matter collected in their layers is compared with typical regions that are unaffected by feedback processes. Numerical simulations have found that the net amount of star formation might be lower for $\mathrm{H}$ II regions, since large portions of neutral material are expected to be dispersed by the ionizing star(s) (Dale et al. 2012; Walch et al. 2012). The overdensity of star-forming clumps near bubbles has been significantly well established (e.g., Deharveng et al. 2010; Hou \& Gao 2014; Kendrew et al. 2016). These observation have given support to the $\mathrm{C} \& \mathrm{C}$ theory as the main physical mechanism responsible for the formation of clumps, where swept-up material is compressed by the expansion, fragments, and forms clumps through gravitational instabilities (Elmegreen \& Lada 1977; Whitworth et al. 1994). However, because we find more cold dust in these locations, it is essential to estimate how star formation processes inside the shells differ from processes in other regions that are unaffected by feedback effects. 
An important quantity to address in this context is therefore to infer how efficiently the cold molecular gas in the shells is converted into star-forming clumps. We do this by estimating the clump formation efficiency (CFE) of the bubbles (Eden et al. 2012, 2013). The CFE can be determined by calculating the ratio between the masses of the bubbles $\left(M_{\mathrm{bub}}\right)$, calculated in Sect. 4.3, and their respective associated Hi-GAL sources (prestellar and protostellar clumps).

To be more consistent, we decided to estimate the envelope mass of the Hi-GAL sources in the same manner as $M_{\text {bub }}$ was calculated (i.e., directly form the Hi-GAL column-density maps) to provide a more reliable estimate of the CFE. In this manner, mass uncertainties that are due to incorrect distance estimates, unreliable SED fits, or inaccurate background subtraction can be significantly attenuated. Envelope masses of Hi-GAL prestellar $\left(M_{\text {pre }}\right)$ and protostellar $\left(M_{\text {pro }}\right)$ clumps located inside bubbles $\left(<R_{\text {shell }}\right)$ were calculated by integrating the column density map over their circularized FWHM sizes ${ }^{7}$. This provided us with estimates of the CFE of prestellar clumps $\left(\mathrm{CFE}_{\mathrm{pre}}=M_{\mathrm{pre}} / M_{\mathrm{bub}}\right)$ and protostellar clumps $\left(\mathrm{CFE}_{\mathrm{pro}}=M_{\text {pro }} / M_{\mathrm{bub}}\right)$ for the sample of 182 bubbles we used to estimate dynamic ages in Sect. 4.2.

We obtained considerably high values of CFEs for both prestellar, $\left\langle\mathrm{CFE}_{\text {pre }}\right\rangle=0.051 \pm 0.030$ and protostellar $\left\langle\mathrm{CFE}_{\text {pro }}\right\rangle=$ $0.102 \pm 0.061$ clumps. The distribution of CFEs is shown in the histogram of Fig. 9. For the total sum of prestellar and protostellar clumps we obtain $\left\langle\mathrm{CFE}_{\text {tot }}\right\rangle=0.148 \pm 0.065$, which means that typically $\sim 15 \%$ of the molecular gas around the bubbles are concentrated in the form of prestellar or protostellar clumps. For comparison, Elia et al. (2013) estimated a CFE 0.02-0.07 for both protostellar and starless (including gravitationally bound and unbound) Hi-GAL clumps in four regions located in the outer part of the Galactic plane $\left(217^{\circ}<l<224^{\circ}\right)$. However, since gravitationally unbound clumps are not included in our analysis, the difference between the CFE that we obtained with the CFE of these four regions is probably higher. Furthermore, Elia et al. estimated the mass of the considered regions using NANTEN CO(1-0) observations, which makes this comparison less reliable.

In order to make a more consistent comparison, we also evaluated the CFE outside the bubbles $\left(\mathrm{CFE}_{\text {out }}\right)$ between $R_{\text {shell }}<r<$ $4 \times R_{\text {eff }}$. This was made by evaluating the ratio between the total amount of integrated column density inside clumps (prestellar and protostellar) located in the outer region and the total integrated area in the outer regions. The dotted color lines in Fig. 9 show that the values of $\mathrm{CFE}_{\text {out }}$ are systematically lower than the CFE calculated for clumps inside the bubble shells, by an average factor of $1.97 \pm 1.12$. Moreover, comparing these values with well-known active star-forming regions, we find that the CFE inside the bubbles is also twice the value obtained for RCW106 (Nguyen et al. 2015) and W43 (Nguyen Luong et al. 2011), and approximately 10 times higher than Cygnus X (Motte et al. 2007).

Provided with estimates of the bubble dynamic ages (Sect. 4.2), we determine possible variations of the CFE with the evolution of bubbles (bottom Fig. 9). Surprisingly, we find that $\mathrm{CFE}_{\text {pro }}$ tends to decrease with the age of the bubble, while $\mathrm{CFE}_{\text {pre }}$ seems to remain nearly constant. A more careful interpretation, however, is required to fully understand the possible reasons for this trend. For instance, we need to consider that at large distances we might on one hand obtain fewer protostellar clumps

\footnotetext{
We convolved the deconvolved clump sizes $\left(F W H M_{\mathrm{dec}}\right)$ provided in the Hi-GAL physical catalog into the resolution of the column density $\operatorname{map}\left(\sqrt{F W H M_{\mathrm{dec}}^{2}+36.3^{\prime \prime 2}}\right)$.
}

because of the sensitivity limit of the Herschel PACS $70 \mu \mathrm{m}$ band, and on the other hand, we might obtain more source confusion with overlapping prestellar clumps (see Elia et al. 2017). Based on Spearman's rank correlation, we find a moderate correlation between the kinematic distances and the estimated dynamic ages (see also Fig. 8) with a coefficient of $r_{\mathrm{s}} \sim 0.46$ and a $p$-value of $\sim 10^{-11}$. Therefore we should consider the possibility that the decreasing trend of $\mathrm{CFE}_{\text {pro }}$ with age could be the result of fewer detections at farther distances that is due to sensitivity. However, we find no evidence for a correlation between the kinematic distances and $\mathrm{CFE}_{\text {pro }}$ when applying the same test $\left(r_{\mathrm{s}} \sim-0.15 ; p\right.$-value $\left.\sim 0.05\right)$, while the correlation between the dynamic ages of the bubble and $\mathrm{CFE}_{\text {pro }}$ seen in Fig. 9 is statistically significant $\left(r_{\mathrm{s}} \sim-0.58 ; p\right.$-value $\left.\sim 10^{-16}\right)$.

Moreover, the different trends between prestellar and protostellar CFEs could be an indirect indication of changes in their formation rate as bubbles expand. A possible interpretation of these different trends might be a faster conversion between the prestellar to protostellar phase for younger bubbles, which conversion gradually decreases as the expansion decelerates and the ionization radiation has a weaker impact. Nevertheless, a detailed individual analysis of these bubbles is required to prove changes in the formation rate between prestellar and protostellar clumps. If the formation rates in H II regions are similar to those of typical star-forming molecular clouds, we expect that the net amount of star formation is higher. We therefore suggest that $\mathrm{H}$ II regions may have a positive impact on the star formation process throughout our Galaxy.

We estimate that $\sim 23 \%$ of the Hi-GAL star-forming clumps ( $\sim 15 \%$ and $\sim 41 \%$ for prestellar and protostellar clumps, respectively) located in the inner part of the Galactic plane are found projected toward a bubble $\left(<R_{\text {shell }}\right)$. We find this value to be consistent with the $\sim 25 \%$ ATLASGAL clumps found by Kendrew et al. (2016) from studying MWP bubbles. It is particularly interesting to note the higher fraction of protostellar clumps. A possible interpretation for the higher number of protostellar objects might be a higher formation rate that converts prestellar clumps into protostellar clumps faster, possibly as a result of a feedback effect (through the C\&C or RDI mechanism). Overall, these numbers suggest that the formation of many stars in the Galaxy may have been triggered by (HII) bubbles.

\subsection{High-mass star formation in bubbles}

Statistical studies of massive MYSOs and their relation to bubbles have been recently made. In particular, Thompson et al. (2012) and Kendrew et al. (2012) presented a statistical study showing that a significant overdensity of MYSOs was identified in the Red MSX Source (RMS) survey (Urquhart et al. 2008), near the rims of bubbles. When we take the typical lifetime of a few $10^{5} \mathrm{yr}$ (Mottram et al. 2011) into account, these object trace very recent star formation. In addition to proposing that MYSOs were triggered by the expansion of (HII) bubbles, both studies found evidence that bubbles associated with RMS MYSOs tend to have smaller angular sizes, which suggests that MYSOs tend to form around younger bubbles.

In order to shed some light into this subject, we studied the potential association of RMS MYSOs with the 182 MWP bubbles for which we have derived the dynamic age. We find that nearly $40 \%$ (71) of these bubbles are associated with at least one MYSO. We then analyzed the average number of MYSOs associated with bubbles $\left(<R_{\text {shell }}\right)$ and searched for possible trends with the dynamic ages of the bubbles (see Fig. 10a). 
Interestingly, we find a higher fraction of younger bubbles $(<2 \mathrm{Myr})$ that are associated with at least one RMS MYSOs and also with higher numbers. Around $50 \%$ of the bubbles younger than 2 Myr are associated with RMS MYSOs (43 out of 86) with an average number of $2.0 \pm 1.1$ MYSOs, while only $29 \%$ of bubbles older than 2 Myr have associated MYSO (28 out of 96) with an average of $1.5 \pm 1.2$ MYSOs.

This is also evident from the average surface density of rms MYSO, using both apparent and physical sizes, as a function of dynamic age in Fig. 10b. This result suggests that the timescale for the formation of MYSOs in bubbles is probably fairly short and very effective at the early stages of the bubble expansion. We note that the RMS source catalog, which is composed of sources with $L>10^{4} L_{\odot}$, is expected to be complete up to distances $\sim 15 \mathrm{kpc}$ (Thompson et al. 2012), which includes all the distances attributed to our subsample of bubbles.

Given the short time of expansion of the bubbles, the question arises as to whether the MYSOs were already present (or undergoing formation) before the formation of the (HII) bubbles or if they had the time to form through gravitational instability of the shell (C\&C process). To search for a potential answer, we estimated the fragmentation time for the shell of these bubbles using the analytical framework of Whitworth et al. (1994), in order to relate the results with the dynamic ages of the bubbles. Following Whitworth et al., the time at which fragmentation starts is determined by the following relation:

$t_{\text {frag }} \sim 1.56 \mathrm{Myr}\left(\frac{c_{\mathrm{s}}}{0.2 \mathrm{~cm} \mathrm{~s}^{-1}}\right)^{\frac{7}{11}}\left(\frac{N_{\mathrm{Lyc}}}{10^{49} \mathrm{~s}^{-1}}\right)^{\frac{-1}{11}}\left(\frac{n_{0}}{100 \mathrm{~cm}^{-3}}\right)^{\frac{-5}{3}}$

where $c_{\mathrm{s}}$ is the uniform isothermal sound speed, $N_{\text {Lyc }}$ is the Lyman-continuum photon emission and $n_{0}$ is the initial mean number density of the medium before the expansion of the $\mathrm{H}$ II region. To obtain an estimate of $n_{0}$, we assumed that the entire estimated mass contained in the shell of bubbles was previously uniformly distributed inside the total volume of the bubbles $\left(\leq R_{\text {eff }}\right)$. This resulted in an average $\left\langle n_{0}\right\rangle \approx 2500 \mathrm{~cm}^{-3}$ ranging from 100 to $1.8 \times 10^{4} \mathrm{~cm}^{-3}$ for our sample of $182 . N_{\text {Lyc }}$ was derived from the NVSS and PMNS radio continuum fluxes (see Sect. 4.2). An isothermal sound speed of the medium ranging from 0.2 to $0.6 \mathrm{~km} \mathrm{~s}^{-1}$ was also assumed.

In Fig. 10c we display the estimated shell fragmentation times against the dynamic ages of the bubbles. Assuming $c_{\mathrm{s}}=$ $0.2 \mathrm{~km} \mathrm{~s}^{-1}$, we find that 132 bubbles $(\sim 73 \%)$ have fragmentation times shorter than their predicted dynamic age. However, we find that the number of bubbles would decrease to $53(\sim 30 \%)$ when $c_{\mathrm{s}}=0.6 \mathrm{~km} \mathrm{~s}^{-1}$ is assumed.

According to this analysis, a significant fraction of these bubbles might not have had time to fragment, therefore we should consider whether the associated MYSOs existed before the bubble began to expand or possibly were already undergoing formation. This question has been discussed before. As an example, Dewangan \& Ojha (2013) performed a detailed study of the N14 bubble (from the Churchwell et al. catalog) and estimated a shorter dynamic age than the predicted fragmentation time. Numerical simulations also show that preexisting dense structures in a fractal medium can further enhance their density through expansion of the bubble, which leads them to collapse faster than they originally would (Walch et al. 2012, 2015). Furthermore, star formation is predicted to occur around $\sim 0.5 \mathrm{Myr}$ after the expansion of the H II region (Walch et al. 2013), which agrees with our observations and dynamic age estimates.
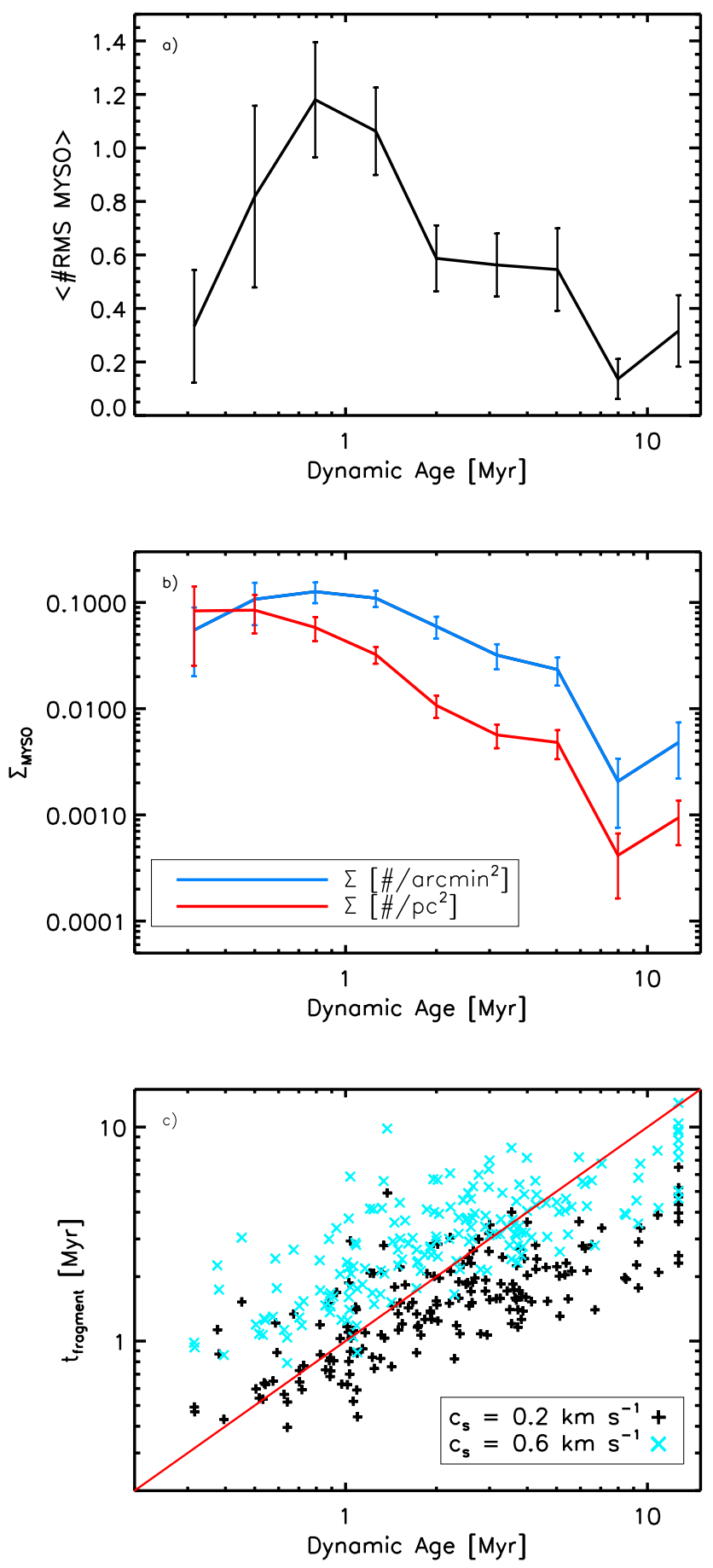

Fig. 10. a) Average number of RMS sources as a function of dynamic age. $b$ ) Average apparent (in blue) and physical (in red) surface densities of RMS sources as a function of dynamic age. The error bars show the standard deviation of the mean $( \pm 1 \sigma / \sqrt{(} N))$. c) Fragmentation time as a function of dynamic time (Whitworth et al. 1994), assuming $c_{\mathrm{s}}=0.2$ (in black) and $0.6 \mathrm{~km} \mathrm{~s}^{-1}$ (in blue). The solid red line represents the one-to-one relation.

These points indicate that the initial environment surrounding an H II region is probably not well described by a neutral medium with uniform density. As discussed in Sect. 5.1, the presence of more evolved YSOs inside bubbles suggests that the medium was actively forming stars at the moment the massive star(s) started to ionize the medium, indicating that density fluctuation were probably present in the medium. Furthermore, 
the high-density environment of the bubbles at early stages of their expansion and their high CFE are favorable physical conditions for a more efficient formation of MYSOs during the earlier stages of bubble expansion ( $<2 \mathrm{Myr}$ ).

We note that a Galactic plane bubble catalog has recently been produced within the VIALACTEA consortium. Bubble identification was obtained based on localized active contours that allow tracing the heated dust of bubble structures seen in emission in the Herschel $70 \mu \mathrm{m}$ images and to define their morphology in the form of contours (Carey et al., in prep.) ${ }^{8}$. The additional information of the morphology of the bubbles and their inhomogeneities will allow us to further improve our understanding of the impact of ionizing stars on the interstellar medium.

\section{Conclusions}

We carried out an extensive statistical study of star-forming objects in the vicinity of Galactic bubbles and analyzed their local environment. The range of evolutionary stages of the starforming objects, from prestellar clumps to Class II YSOs, provided a clear and distinct picture of the spatial distribution of star-forming sources in the surroundings of 1360 (HII) bubbles. Prestellar and protostellar clumps were taken from the Hi-GAL catalog, while YSO candidates were classified using Spitzer/IRAC fluxes. This led to a total of $\sim 70000$ star-forming objects that were compiled into surface density maps. These revealed a clear evolutionary trend, where more evolved sources (Class II YSO candidates) are mostly spatially found concentrated near the center of the bubble, while younger sources (HIGAL prestellar and protostellar clumps) are found at the rim. We find $80 \%$ more sources per unit area in the direction of the bubbles $\left(<R_{\text {shell }}\right)$ than outside the bubbles $\left(R_{\text {shell }}<r<4 R_{\text {eff }}\right)$. We evaluated and excluded the possibility of potential biases due to the YSO classification method by using different classification methods. Furthermore, YSO candidates from a recent WISE catalog (Marton et al. 2016) also show a clear overdensity of YSO sources toward the inner part of the bubbles, strengthening this result.

We were able to determine the dynamic age for a subsample of 182 bubbles for which we found available kinematic distances and radio continuum flux measurements. Most of the bubbles $(\sim 80 \%)$ have a younger dynamic age than $4 \mathrm{Myr}$, which is comparable to the lifetime of an O5 star. Most of the bubbles are located in the spiral arms identified by Russeil (2003). The shell density of the bubbles, derived form the Hi-GAL column density maps, show a decreasing trend with dynamic ages, suggesting that the bubbles are formed in high-density regions. We argued that the shell density tends to decrease with time as a result of the expansion of the shell volume and is presumably due to the conversion of the shell mass into stars.

We estimate that the CFE of the Hi-GAL clumps located in the bubbles $\left(<R_{\text {shell }}\right)$ is $\sim 15 \%$, which is approximately twice higher than the clumps located outside $\left(R_{\text {shell }}<r<4 R_{\text {eff }}\right)$ and is also higher than the CFE reported in other known active starforming regions. We find, however, that the CFE is higher for protostellar than prestellar clumps. In particular, the CFE of protostellar clumps has a wider spread of values that are higher for younger bubbles and decrease with dynamic ages of the bubbles. We interpret this trend as a possible increase in the formation rate from the prestellar to protostellar phase at the early stages

\footnotetext{
8 The active contour algorithm has been developed in the University of Leeds.
}

of the bubble expansion, which would eventually decrease as the impact of the expansion and the ionization weakens.

The fraction of clumps that is spatially associated with bubbles is $\sim 23 \%$, consistent with the fraction of ATLASGAL clumps in the vicinity of MWP bubbles (Kendrew et al. 2016). However, for the individual fraction of protostellar clumps we obtain $41 \%$. We argue that the higher fraction of protostellar clumps may be related with the higher protostellar clump formation rate in bubbles.

Bubbles younger than $2 \mathrm{Myr}$ on average have more RMS MYSOs, and more of them are associated with at least one MYSO than in older bubbles. This may be related to the higher density of shells in younger bubbles, which is necessary to form MYSOs. We discussed whether the MYSOs have had time to form through gravitational instability of the shell or whether another physical mechanism may be at play. The shell fragmentation times estimated for this purpose (following Whitworth et al. 1994) showed that a significant fraction of bubble shells would not have had time to fragment. We therefore argued that dense structures existed in the medium before the bubble expansion to allow for a more comparable star-formation timescale, as shown in the simulations performed in Walch et al. $(2012,2015)$.

The results reported here point toward the possibility that the YSOs we found inside the bubbles were probably undergoing their formation process before the feedback from the ionizing source(s). On the other hand, Hi-GAL clumps and RMS MYSOs, which trace more recent star-formation activity, indicate that these object were probably triggered in the shell of the bubbles through a combination of C\&C and RDI processes. Furthermore, the high fraction and CFE of protostellar clumps in the early stages of the bubble expansion where the density of the shell is higher might indicate an acceleration of the star-formation process through the feedback effect of the (HII) bubbles.

Acknowledgements. This work is part of the VIALACTEA Project, a Collaborative Project under Framework Programme 7 of the European Union, funded under Contract \#607380, which is hereby acknowledged. Herschel Hi-GAL data processing, map production, and source catalog generation is the result of a multi-year effort that was initially funded thanks to Contracts I/038/080/0 and I/029/12/0 from ASI, Agenzia Spaziale Italiana. Pedro Palmeirim acknowledges support from the Fundação para a Ciência e a Tecnologia of Portugal (FCT) through national funds (UID/FIS/04434/2013) and by FEDER through COMPETE2020 (POCI-01-0145-FEDER-007672) and also by the fellowship SFRH/BPD/110176/2015 funded by FCT (Portugal) and POPH/FSE (EC). Herschel is an ESA space observatory with science instruments provided by European-led Principal Investigator consortia and with important participation from NASA. This work is based on observations obtained with Herschel-PACS and Herschel-SPIRE photometers. PACS has been developed by a consortium of institutes led by MPE (Germany) and including UVIE (Austria); KU Leuven, CSL, IMEC (Belgium); CEA, LAM (France); MPIA (Germany); INAFIFSI/OAA/OAP/OAT, LENS, SISSA (Italy); IAC (Spain). This development has been supported by the funding agencies BMVIT (Austria), ESA-PRODEX (Belgium), CEA/CNES (France), DLR (Germany), ASI/INAF (Italy), and CICYT/MCYT (Spain). SPIRE has been developed by a consortium of institutes led by Cardiff Univ. (UK) and including: Univ. Lethbridge (Canada); NAOC (PR China); CEA, LAM (France); IFSI, Univ. Padua (Italy); IAC (Spain); Stockholm Observatory (Sweden); Imperial College London, RAL, UCL-MSSL, UKATC, Univ. Sussex (UK); and Caltech, JPL, NHSC, Univ. Colorado (USA). This development has been supported by national funding agencies: CSA (Canada); NAOC (China); CEA, CNES, CNRS (France); ASI (Italy); MCINN (Spain); SNSB (Sweden); STFC, UKSA (UK); and NASA (USA). This work is based on observations made with the Spitzer Space Telescope, which is operated by the Jet Propulsion Laboratory, California Institute of Technology, under contract with NASA. We have made use of the NASA/IPAC Infrared Science Archive to obtain data products from the 2MASS, Spitzer-GLIMPSE, and Spitzer-MIPSGAL surveys. This publication has been made possible by the participation of more than 35000 volunteers on the Milky Way Project. Their contributions are acknowledged individually at http://www . milkywayproject.org/authors. 


\section{References}

Allen, C. W. 1973, Astrophysical Quantities (Athlone Press)

Allen, L. E., Calvet, N., D’Alessio, P., et al. 2004, ApJS, 154, 363

Anderson, L. D., Bania, T. M., Balser, D. S., \& Rood, R. T. 2011, ApJS, 194, 32

Anderson, L. D., Bania, T. M., Balser, D. S., et al. 2014, VizieR Online Data Catalog, J/ApJS/212/1

Balser, D. S., Rood, R. T., Bania, T. M., \& Anderson, L. D. 2011, ApJ, 738, 27

Bergin, E. A., \& Tafalla, M. 2007, ARA\&A, 45, 339

Bernard, J.-P., Paradis, D., Marshall, D. J., et al. 2010, A\&A, 518, L88

Cesarsky, D., Jones, A. P., Lequeux, J., \& Verstraete, L. 2000, A\&A, 358, 708

Churchwell, E., Povich, M. S., Allen, D., et al. 2006, ApJ, 649, 759

Churchwell, E., Watson, D. F., Povich, M. S., et al. 2007, ApJ, 670, 428

Condon, J. J., Cotton, W. D., Greisen, E. W., et al. 1998, AJ, 115, 1693

Cutri, R. M., et al. 2013, VizieR Online Data Catalog, II/328

Dale, J. E., Ercolano, B., \& Bonnell, I. A. 2012, MNRAS, 427, 2852

Dale, J. E., Haworth, T. J., \& Bressert, E. 2015, MNRAS, 450, 1199

Deharveng, L., Zavagno, A., \& Caplan, J. 2005, A\&A, 433, 565

Deharveng, L., Schuller, F., Anderson, L. D., et al. 2010, A\&A, 523, A6

Deharveng, L., Zavagno, A., Samal, M. R., et al. 2015, A\&A, 582, A1

Desert, F.-X., Boulanger, F., \& Puget, J. L. 1990, A\&A, 237, 215

Dewangan, L. K., \& Ojha, D. K. 2013, MNRAS, 429, 1386

Draine, B. T., \& Li, A. 2007, ApJ, 657, 810

Dyson, J. E., \& Williams, D. A. 1980, Physics of the Interstellar Medium

Eden, D. J., Moore, T. J. T., Plume, R., \& Morgan, L. K. 2012, MNRAS, 422, 3178

Eden, D. J., Moore, T. J. T., Morgan, L. K., Thompson, M. A., \& Urquhart, J. S. 2013, MNRAS, 431, 1587

Egan, M. P., Price, S. D., Kraemer, K. E., et al. 2003, VizieR Online Data Catalog, V/114

Elia, D., Schisano, E., Molinari, S., et al. 2010, A\&A, 518, L97

Elia, D., Molinari, S., Fukui, Y., et al. 2013, ApJ, 772, 45

Elia, D., Molinari, S., Schisano, E., et al. 2017, MNRAS, 471, 100

Elmegreen, B. G., \& Lada, C. J. 1977, ApJ, 214, 725

Evans, II, N. J., Dunham, M. M., Jørgensen, J. K., et al. 2009, ApJS, 181, 321

Fang, M., van Boekel, R., King, R. R., et al. 2012, A\&A, 539, A119

Gaczkowski, B., Preibisch, T., Ratzka, T., et al. 2013, A\&A, 549, A67

Geen, S., Hennebelle, P., Tremblin, P., \& Rosdahl, J. 2015, MNRAS, 454, 4484

Greene, T. P., Wilking, B. A., Andre, P., Young, E. T., \& Lada, C. J. 1994, ApJ, 434, 614

Griffin, M. J., Abergel, A., Abreu, A., et al. 2010, A\&A, 518, L3

Gritschneder, M., \& Burkert, A. 2014, MNRAS, 438, 1318

Gutermuth, R. A., \& Heyer, M. 2015, AJ, 149, 64

Gutermuth, R. A., Myers, P. C., Megeath, S. T., et al. 2008, ApJ, 674, 336

Gutermuth, R. A., Megeath, S. T., Myers, P. C., et al. 2009, ApJS, 184, 18

Heiderman, A., Evans, II, N. J., Allen, L. E., Huard, T., \& Heyer, M. 2010, ApJ, 723,1019

Hester, J. J., Scowen, P. A., Sankrit, R., et al. 1996, AJ, 111, 2349

Hou, L. G., \& Gao, X. Y. 2014, MNRAS, 438, 426

Indebetouw, R., Mathis, J. S., Babler, B. L., et al. 2005, ApJ, 619, 931

Kauffmann, J., Bertoldi, F., Bourke, T. L., Evans, II, N. J., \& Lee, C. W. 2008, A\&A, 487, 993

Kendrew, S., Simpson, R., Bressert, E., et al. 2012, ApJ, 755, 71

Kendrew, S., Beuther, H., Simpson, R., et al. 2016, ApJ, 825, 142

Kumar, M. S. N., Keto, E., \& Clerkin, E. 2006, A\&A, 449, 1033

Lada, C. J. 1987, in Star Forming Regions, eds. M. Peimbert, \& J. Jugaku, IAU Symp., 115,1

Lada, C. J., Muench, A. A., Luhman, K. L., et al. 2006, AJ, 131, 1574

Larson, R. B. 1981, MNRAS, 194, 809

Leger, A., \& Puget, J. L. 1984, A\&A, 137, L5

Liu, H.-L., Wu, Y., Li, J., et al. 2015, ApJ, 798, 30

Liu, H.-L., Li, J.-Z., Wu, Y., et al. 2016, ApJ, 818, 95

Lu, J. R., Neichel, B., Anderson, J., et al. 2014, in Adaptive Optics Systems IV, Proc. SPIE, 9148, 91480B

Martín-Hernández, N. L., Vermeij, R., \& van der Hulst, J. M. 2005, A\&A, 433, 205

Martins, F., Pomarès, M., Deharveng, L., Zavagno, A., \& Bouret, J. C. 2010, A\&A, 510, A32

Marton, G., Tóth, L. V., Paladini, R., et al. 2016, MNRAS, 458, 3479

McKee, C. F., \& Ostriker, E. C. 2007, ARA\&A, 45, 565

Molinari, S., Swinyard, B., Bally, J., et al. 2010, A\&A, 518, L100

Molinari, S., Schisano, E., Elia, D., et al. 2016, A\&A, 591, A149

Motte, F., Bontemps, S., Schilke, P., et al. 2007, A\&A, 476, 1243

Mottram, J. C., Hoare, M. G., Urquhart, J. S., et al. 2011, A\&A, 525, A149

Natoli, P., de Gasperis, G., Gheller, C., \& Vittorio, N. 2001, A\&A, 372, 346

Neichel, B., Samal, M. R., Plana, H., et al. 2015, A\&A, 576, A110
Nguyen, H., Nguyen-Luong, Q., Martin, P. G., et al. 2015, ApJ, 812, 7 Nguyen Luong, Q., Motte, F., Schuller, F., et al. 2011, A\&A, 529, A41 Ott, S. 2010, in Astronomical Data Analysis Software and Systems XIX, eds. Y. Mizumoto, K.-I. Morita, \& M. Ohishi, ASP Conf. Ser., 434, 139

Paladini, R., Umana, G., Veneziani, M., et al. 2012, ApJ, 760, 149

Pezzuto, S., Elia, D., Schisano, E., et al. 2012, A\&A, 547, A54

Pilbratt, G. L., Riedinger, J. R., Passvogel, T., et al. 2010, A\&A, 518, L1

Poglitsch, A., Waelkens, C., Geis, N., et al. 2010, A\&A, 518, L2

Povich, M. S., Churchwell, E., Bieging, J. H., et al. 2009, ApJ, 696, 1278

Povich, M. S., Kuhn, M. A., Getman, K. V., et al. 2013, ApJS, 209, 31

Rebull, L. M., Padgett, D. L., McCabe, C.-E., et al. 2010, ApJS, 186, 259

Rigaut, F., Neichel, B., Boccas, M., et al. 2014, MNRAS, 437, 2361

Robitaille, T. P., Meade, M. R., Babler, B. L., et al. 2008, AJ, 136, 2413

Russeil, D. 2003, A\&A, 397, 133

Russeil, D., Zavagno, A., Motte, F., et al. 2010, A\&A, 515, A55

Samal, M. R., Zavagno, A., Deharveng, L., et al. 2014, A\&A, 566, A122

Schisano, E., Rygl, K. L. J., Molinari, S., et al. 2014, ApJ, 791, 27

Simpson, R. J., Povich, M. S., Kendrew, S., et al. 2012, MNRAS, 424, 2442

Spitzer, L. 1978, Physical Processes in the Interstellar Medium (New York: Wiley-Interscience)

Spitzer Science Center 2009, VizieR Online Data Catalog, II/293

Stern, D., Eisenhardt, P., Gorjian, V., et al. 2005, ApJ, 631, 163

Strafella, F., Lorenzetti, D., Giannini, T., et al. 2015, ApJ, 798, 104

Thompson, M. A., Urquhart, J. S., Moore, T. J. T., \& Morgan, L. K. 2012, MNRAS, 421, 408

Tielens, A. G. G. M. 2008, ARA\&A, 46, 289

Traficante, A., Calzoletti, L., Veneziani, M., et al. 2011, MNRAS, 416, 2932

Tremblin, P., Audit, E., Minier, V., Schmidt, W., \& Schneider, N. 2012, A\&A, 546, A33

Tremblin, P., Schneider, N., Minier, V., et al. 2014, A\&A, 564, A106

Urquhart, J. S., Hoare, M. G., Lumsden, S. L., Oudmaijer, R. D., \& Moore, T. J. T. 2008, in Massive Star Formation: Observations Confront Theory, eds. H. Beuther, H. Linz, \& T. Henning, ASP Conf. Ser., 387, 381

Walch, S., Whitworth, A. P., \& Girichidis, P. 2012, MNRAS, 419, 760

Walch, S., Whitworth, A. P., Bisbas, T. G., Wünsch, R., \& Hubber, D. A. 2013, MNRAS, 435, 917

Walch, S., Whitworth, A. P., Bisbas, T. G., Hubber, D. A., \& Wünsch, R. 2015, MNRAS, 452, 2794

Whitworth, A. P., Bhattal, A. S., Chapman, S. J., Disney, M. J., \& Turner, J. A. 1994, MNRAS, 268, 291

Wright, A. E., Griffith, M. R., Burke, B. F., \& Ekers, R. D. 1994, ApJS, 91, 111

Wright, E. L., Eisenhardt, P. R. M., Mainzer, A. K., et al. 2010, AJ, 140, 1868

Zavagno, A., Pomarès, M., Deharveng, L., et al. 2007, A\&A, 472, 835

Zavagno, A., Russeil, D., Motte, F., et al. 2010, A\&A, 518, L81

Zinnecker, H., \& Yorke, H. W. 2007, ARA\&A, 45, 481

1 Aix Marseille Univ., CNRS, LAM, Laboratoire d'Astrophysique de Marseille, Marseille, France e-mail: pedro.palmeirim@lam. fr

2 Instituto de Astrofísica e Ciências do Espaço, Universidade do Porto, CAUP, Rua das Estrelas, 4150-762 Porto, Portugal e-mail: pedro.palmeirim@astro.up.pt

3 Istituto di Astrofisica e Planetologia Spaziali INAF-IAPS, via Fosso del Cavaliere 100, 00133 Roma, Italy

${ }^{4}$ Astrophysics Research Institute, Liverpool John Moores University, Liverpool Science Park Ic2, 146 Brownlow Hill, Liverpool L3 5RF, UK

5 School of Physics \& Astronomy, Cardiff University, Cardiff, CF24, 3AA, UK

${ }^{6}$ Maison de la Simulation, CEA-CNRS-UPS-UVSQ, USR 3441, Centre d'Étude de Saclay, 91191 Gif- Sur-Yvette, France

7 Université de Strasbourg, CNRS, Observatoire Astronomique de Strasbourg, UMR 7550, 67000 Strasbourg, France

8 CERN, Route de Meyrin 385, 1217 Meyrin, Switzerland

${ }^{9}$ INAF-Osservatorio Astrofisico di Catania, via S. Sofia 78, 95123 Catania, Italy

10 School of Physics and Astronomy, University of Leeds, Leeds, UK

11 INAF-Osservatorio Astronomico di Trieste, via G. B. Tiepolo 11, Trieste, Italy

12 MTA-SZTAKI, 1111 Budapest, Kende u. 13-17, Hungary

13 Space Telescope Science Institue, 3700 San Martin Drive, Baltimore MD, USA 


\section{Appendix A: additional figures}
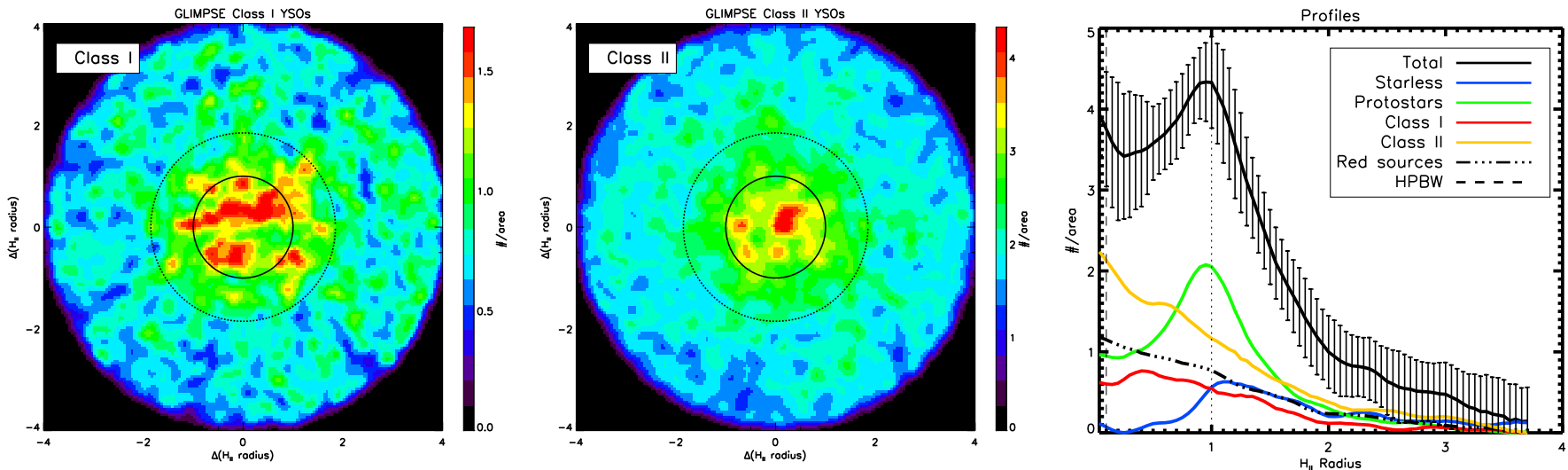

Fig. A.1. Same as Figs. 3 and 4, preformed for the YSO sample obtained by exempting quality detection at the IRAC [8.0] band and using the MIPS [24] photometric measurement in the YSO classification when available.

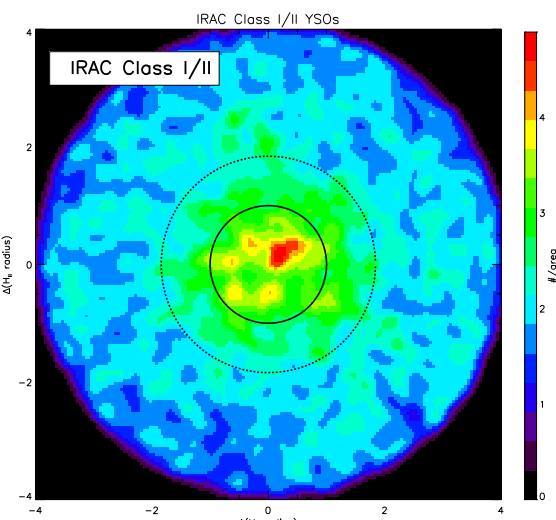

$\triangle\left(H_{1}\right.$ rodius $)$

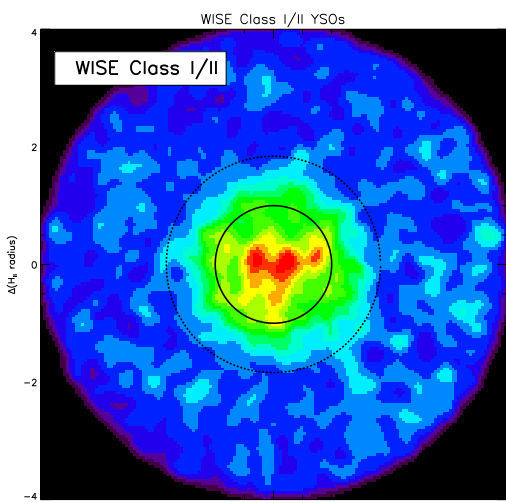

$\Delta\left(H_{n}\right.$ ododis $)$

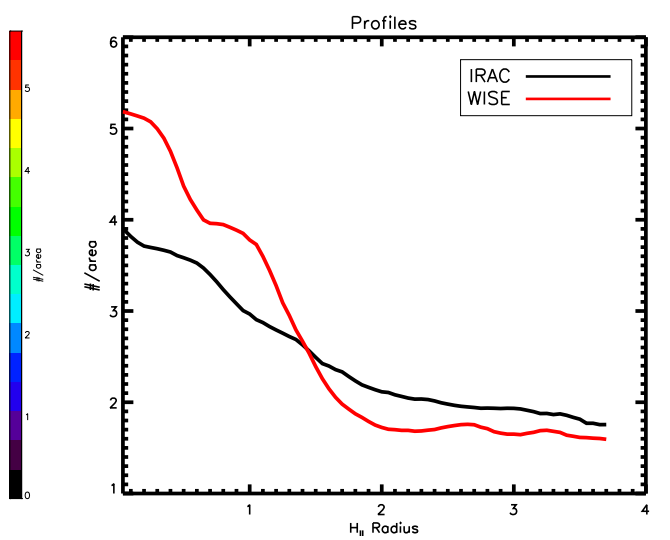

Fig. A.2. Surface density maps for all Class I and II candidates classified using the four IRAC flux criteria in Sect. 3.2 (left) and candidates taken from the WISE catalog from Marton et al. (2016) (center). The spatial scales of the maps are normalized by the bubble radius (solid black circle). The dashed black circle represents the average shell radius $\left(R_{\text {shell }}=1.85 \times R_{\text {eff }}\right)$. The mean radial source density profiles $($ right $)$ for the IRAC YSO candidates (solid black) and WISE YSO candidates (solid red) are shown as a function of distance to the central position of the H II regions, obtained by azimuthally averaging from the surface density maps. 